\title{
Stochastic multiscale model of MEMS stiction accounting for high order statistical moments of non-Gaussian contacting surfaces
}

\author{
T.V. Hoang, L. Wu, J.-C. Golinval, M. Arnst, L. Noels
}

\begin{abstract}
Stiction is a failure mode of microelectromechanical systems (MEMS) involving permanent adhesion of moving surfaces. Models of stiction typically describe the adhesion as a multiple asperity adhesive contact between random rough surfaces, and they thus require a sufficiently accurate statistical representation of the surface, which may be non-Gaussian. If the stiction is caused primarily by multiple asperity adhesive contact in only a small portion of the apparent area of the contacting surfaces, the number of adhesive contacts between asperities may not be sufficiently statistically significant for a homogenized model to be representative. In [Hoang et al., A computational stochastic multiscale methodology for MEMS structures involving adhesive contact, Tribology International, 110:401-425, 2017], the authors have proposed a probabilistic multiscale model of multiple asperity adhesive contact that can capture the uncertainty in stiction behavior. Whereas the previous paper considered Gaussian random rough surfaces, the aim of the present paper is to extend this probabilistic multiscale model to non-Gaussian random rough surfaces whose probabilistic representation accounts for the high order statistical moments of the surface height. The probabilistic multiscale model thus obtained is validated by means of a comparison with experimental data of stiction tests of cantilever beams reported in the literature.
\end{abstract}

Index Terms-multiscale contact, stochastic, maximum entropy principle, adhesion, capillary, van der Waals

\section{INTRODUCTION}

Due to the reduced size of microelectromechanical systems (MEMS), the surface effect is an important factor to be considered in their design. Indeed, between two contacting surfaces, there naturally exist adhesive forces, e.g. van der Waals (vdW) and capillary forces, which can lead to the failure of the involved structures when their surfaces are unexpectedly stuck together [1], [2], [3], [4]. That phenomenon is well-known as the stiction failure. Many experiments were conducted to investigate the stiction phenomenon. For instance, in [5], [6], [7], [8], the stiction tests were implemented by considering micro cantilever beam structures. These experiments were implemented to measure the shortest length of the noncontacting zone (the crack length) for each failure beam, see Fig. 1(a), and then evaluate the apparent adhesion energy. The experimental results showed that the adhesion energies between rough surfaces are smaller than the theoretical ones

This work is supported by a FRIA fellowship of the Belgian National Fund for Education and Research in Industry and Farming.

The authors are with the Department of Aerospace and Mechanical Engineering, University of Liège, Allée de la Découverte 9, Quartier Polytech 1, B4000 Liège, Belgium. (e-mail: vinhvinhtruong@gmail.com, L.Wu@ulg.ac.be, JC.Golinval@ulg.ac.be, Maarten.Arnst@ulg.ac.be, L.Noels@ulg.ac.be).

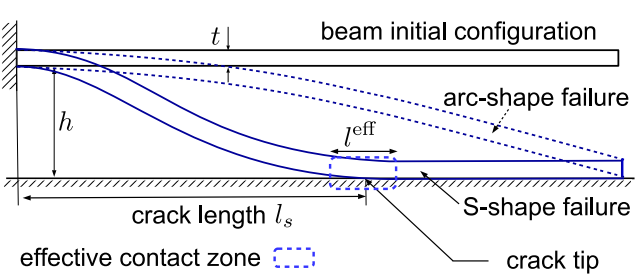

(a)

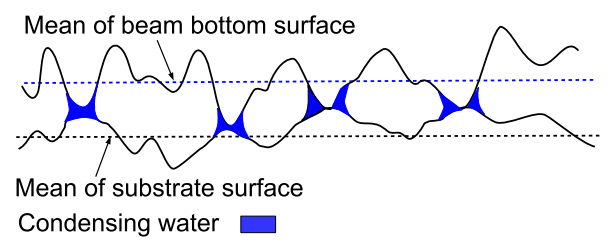

(b) .

Figure 1. The stiction failure of a cantilever beam structure under humid condition: (a) the normal configuration, the arc-shape and S-shape failure configurations (not on scale); and (b) zoom onto a part of the contact zone, which spans from the crack tip to the beam free end, with illustration of the condensing water area on the surface topology (not on scale).

by ratios in the range from $10^{-5}$ to 1 . This range is also the approximation of the ratio between the effective interacting area-the area on which the adhesive stress is applied e.g. the condensing water area in the case of capillary forces-and the apparent one, see Fig. 1(b). These experimental results confirm the multiple-asperity contact theory: due to the roughness of the contacting surfaces, the contact interaction involves only the highest asperities of the surface topology, and the total interacting area is consequently much smaller than the apparent one, as illustrated in Fig. 1(b) [9], [10], [11], [12], [13]. In the context of contact between rough surfaces, the surface topology is usually assumed as a stationary Gaussian random field, e.g. in the Greenwood-Williamson $(\mathrm{GW})$ contact model [9]. With that assumption, the surfaces are modeled by spherical asperities that all have an identical radius but whose heights follow a Gaussian distribution [10]. The contact forces are evaluated by applying the appropriate analytical contact theory, e.g. Hertz [14], DMT [15], JKR [16], or Maugis [17], on these spherical asperities. The integration of these analytical contact theories is achieved at a negligible computational effort which is the main advantage of the GW model in comparison with full numerical methods, such as finite element (FE) methods [18], [19], [20], [21], [22], [23] and molecular dynamics 
(MD) methods [24], [25]. Nevertheless, a comparison between experiments and numerical predictions reported in [5] shows that higher-order statistical moments including skewness and kurtosis of the first order marginal probability density function (mPDF) should be accounted for to improve the accuracy of the numerical predictions.

Moreover, the stiction failure suffers from uncertainty as it was stated in [26]: “... why one MEMS device sticks and another identical one does not". One source of uncertainty originates in the short range property of adhesive stress which is applied on rough contacting surfaces. Due to the short range of adhesive stress (nanometers), the effective contact zone governing the phenomenon is limited around the crack tip [6] in zone $l^{\text {eff }}$, see Fig. 1(a), and the number of contacting asperities involved in that effective contact zone might not be sufficient for deriving a homogenized contact behavior. Therefore, different cantilever beams fabricated with an identical process and using an identical design could end up after the stiction test with different configurations (free, or failure configurations, see Fig. 1(a)), as experimentally observed in [5], [27], [28], [29].

Although the uncertainty characteristics [30], [31], [27], [26] and the non-Gaussianity of the surface height distribution [5], [32], [33] play important roles in the stiction phenomenon, they were either both neglected or accounted for but individually. On the one hand, in [5], the importance of non-Gaussian properties including the skewness and the kurtosis of the contacting surface heights was illustrated by a comparison between numerical predictions and experimental results; however, only deterministic predictions were given. On the other hand, in [30], the authors have developed a stochastic multiscale methodology to predict the uncertainty of the stiction phenomenon but only for Gaussian contacting surfaces.

In this paper, we enhance the stochastic multiscale method developed in [30] by accounting for skewness and kurtosis when simulating the surface roughness. Numerical predictions are compared with experimental results from [5] for the purpose of model validation.

The randomness of the contacting rough surface is first characterized and simulated. Measurements of the contacting surfaces, e.g. by means of atomic-force microscopy (AFM), are usually the input data for this task. In this paper, since we do not have direct access to AFM measurements of the contacting surfaces, we exploit the processed data from [5] consisting of the first few statistical moments of the first order mPDF (variance, skewness, and kurtosis) and the summit statistical properties (mean summit radius and summit density). We then apply the maximum entropy (ME) method, which selects the distribution that maximizes the entropy under the constraints defined by the available information, e.g. statistical moments. We use the remainder of the available information, i.e. the summit statistical properties, to infer the PSD function. In order to conduct Monte Carlo simulations, a surface generator is required. In this paper, the non-Gaussian random surface is constructed as an appropriate transformation of an underlying Gaussian random surface as in [34], [35]; an advantage of this construction is that realizations of the underlying Gaussian

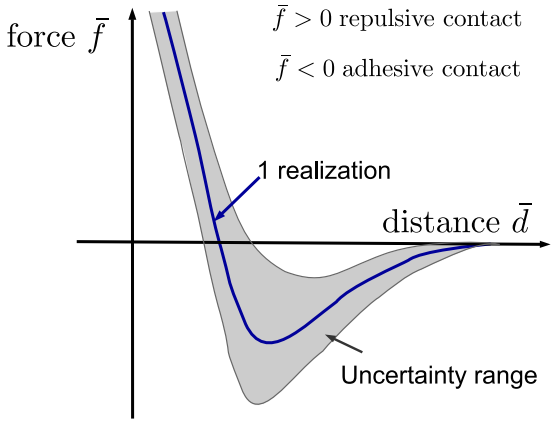

Figure 2. The random apparent contact forces between two rough surfaces.

random surface can be efficiently generated by using spectral representation methods [36].

The semi-analytical adhesive contact model that we have proposed in [31] is then applied to the statistically independent generated surfaces in order to obtain a set of samples of random apparent adhesive contact forces, as illustrated in Fig. 2. This adhesive contact model, motivated by the multiple asperity contact theory, identifies the contacting asperities directly from the generated surfaces, approximates them by spheres and then applies the analytical asperity contact model to evaluate the forces. In contrast to the analytical surface contact models, e.g. GW model, the semi-analytical model can capture the size effect and the uncertainties it induces since the size of the generated surfaces is controlled.

The stochastic model-based multi-scale framework, which was developed by the authors in [30], is then applied. The method consists in constructing a FE model of the considered MEMS structure, in which the random adhesive contact forces are integrated as random contact laws. By performing a Monte Carlo simulation on this model, we evaluate its probabilistic behaviors. However, in order to reduce the computational cost inherent to the evaluation of a large number of contact forces, a stochastic model of the adhesive contact forces is constructed by applying data modeling techniques.

The methodology is developed in two phases, see Fig. 3: (i) the characterization and simulation of contacting surfaces are described in Sec. II, and (ii) the propagation of this randomness using the stochastic model-based multi-scale method is described in Sec. III. In Sec. IV the methodology is implemented for the cantilever beam stiction tests reported in [5]. In Sec. V, the main objectives of this paper including: the effects of the non-Gaussian distribution of the contact surfaces, the uncertainty in the involved structural behaviors, and the validation of the developed method, are reviewed. In addition, owing to the numerical model, a sensitivity of the surface statistical moments on the adhesion energy is analyzed, and the uncertainty of the structural behaviors due to different sources, i.e. from the rough contacting surfaces and from the geometrical dimensions, are compared. Finally, conclusions are given in Sec. VI. 


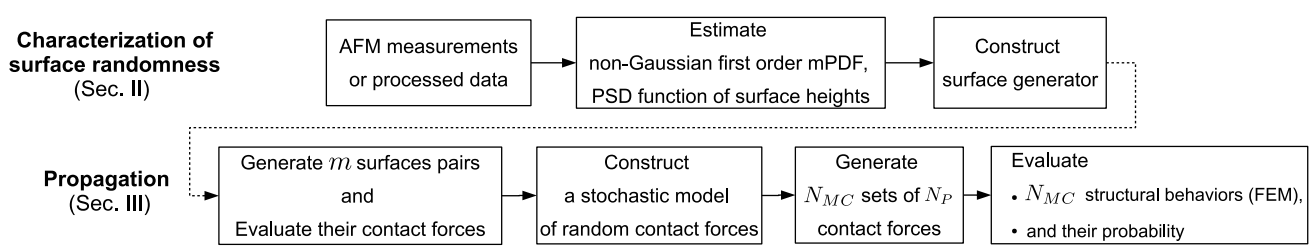

Figure 3. The overview of the methodology including the characterization and simulation of random surfaces, discussed in Sec. II, and the propagation of surface randomness through the stochastic multi-scale model discussed in Sec. III. $N_{M C}$ is the number of MCS and $N_{P}$ is the number of integration points of the FE model, see Sec. III for details.

\section{RANDOMNESS OF SURFACES: CHARACTERIZATION AND SIMULATION}

As illustrated in Fig. 3, the first step of our methodology consists in the characterization and the simulation of the contacting surfaces. We model a random rough surface as a non-Gaussian random field. Specifically, we use the method introduced in [34] for the characterization and the simulation of this non-Gaussian random field. This method constructs the non-Gaussian random field as a nonlinear transformation of an underlying stationary Gaussian random field. Since the method requires the first order mPDF and the PSD function of the nonGaussian random field to be prescribed, we deduce the first order $\mathrm{mPDF}$ from the variance, the skewness, and the kurtosis of the surface heights using the ME method, and we deduce the PSD function from the summit statistical properties.

This section is developed as follows. The estimation of the first order mPDF is explained in Sec. II-A. The surface generator for non-Gaussian cases is described in Sec. II-B from the obtained first order MPDF and a given PSD function. The construction of the PSD function from summit statistical properties is eventually developed in Sec. II-C.

\section{A. Estimation of the first order $m P D F$}

The first four statistical moments $m_{i}$-with $i$ the moment order-of the first order $\mathrm{mPDF} \mathrm{p}_{Z}$ of the non-Gaussian random field $Z(\mathbf{x})$ representing the surface height read as

$$
m_{i}=\int_{\mathfrak{R}} z^{i} \mathrm{p}_{Z}(z) \mathrm{d} z, \text { with } i=1, \cdots, 4 .
$$

The first two moments are the mean-modeled as zero-and the variance. Normalizing the third and forth order moments, we obtain the skewness $\gamma$, and the kurtosis $\beta$ respectively as

$$
\gamma=\frac{m_{3}}{r m s^{3}}, \quad \beta=\frac{m_{4}}{r m s^{4}}
$$

where rms $=\sqrt{m_{2}}(<+\infty)$ is the root mean square roughness. In the case of a Gaussian random field, $\gamma=0$ and $\beta=3$. From these moments, using the ME principle, the first order mPDF of the surface heights can be identified to ensure the same statistical moments. The solution is given by [37]

$$
\mathrm{p}_{Z}(z)=\exp \left(-\lambda_{0}-\sum_{i=1}^{4} \lambda_{i} z^{i}\right)
$$

where $\lambda_{0}, \cdots, \lambda_{4}$ are five Lagrange parameters to be identified, see details in [38], [39].

\section{B. Surfaces generator}

A surface generator is constructed in this section. The input data for the generator is the non-Gaussian first order mPDF $\mathrm{p}_{Z}$ and the PSD function $s_{Z}(\boldsymbol{\zeta})$ with $\zeta=\left\{\zeta_{1}, \zeta_{2}\right\} \in \mathfrak{R}^{2}$ the wave numbers. While $\mathrm{p}_{Z}$ is obtained using the ME method as discussed in Sec. II-A, $s_{Z}$ will be estimated from summit statistical properties in Sec. II-C where the surface generator developed in this section is required. The case of Gaussian surface generator is firstly described before dealing with the more complicated case of non-Gaussian surfaces.

1) Generator of Gaussian surfaces: For Gaussian random fields, the surface generator is constructed for a prescribed PSD function $s_{Z_{\mathrm{G}}}$ following the works of Shinozuka [34] and Poiron and Soize [36]. For a chosen value of the maximum wave number $\mu$, the normal height $\tilde{z}$ can be generated as

$$
\begin{aligned}
\tilde{z}_{\mathrm{G}}(\mathbf{x})=\sqrt{2 \Delta \zeta^{2}} \mathfrak{R e}[ & \sum_{l_{1}=1}^{\mu} \sum_{l_{2}=1}^{\mu} \beta_{\left(l_{1}, l_{2}\right)} \sqrt{\frac{1}{(2 \pi)^{2}} s_{Z_{\mathrm{G}}}\left(\zeta_{l_{1}}, \zeta_{l_{2}}\right)} \\
& \left.\exp \left(\mathrm{i} x_{1} \zeta_{l_{1}}+\mathrm{i} x_{2} \zeta_{l_{2}}+\mathrm{i} \phi_{\left(l_{1}, l_{2}\right)}\right)\right]
\end{aligned}
$$

where

- the values $\left\{\left(\zeta_{l_{1}}, \zeta_{l_{2}}\right), \quad 1 \leq l_{1}, l_{2} \leq \mu\right\}$ are samplings of the wave number domain such that $\left(\zeta_{l_{1}}, \zeta_{l_{2}}\right)=\left(-\zeta_{L}+\right.$ $\left.\left(l_{1}-1\right) \Delta \zeta,-\zeta_{L}+\left(l_{2}-1\right) \Delta \zeta\right)$, with $\Delta \zeta=2 \zeta_{L} / \mu$;

- the values $\left\{\phi_{\left(l_{1}, l_{2}\right)}, 1 \leq l_{1}, l_{2} \leq \mu\right\}$ are $\mu \times \mu$ independent realizations of a uniform random variable with values in $[0,2 \pi]$; and

- the values $\left\{\beta_{\left(l_{1}, l_{2}\right)}, 1 \leq l_{1}, l_{2} \leq \mu\right\}$ are such that $\beta_{\left(l_{1}, l_{2}\right)}=\sqrt{-\log \left(\psi_{\left(l_{1}, l_{2}\right)}\right)}$, where $\left\{\psi_{\left(l_{1}, l_{2}\right)}, \quad 1 \leq\right.$ $\left.l_{1}, l_{2} \leq \mu\right\}$ are $\mu \times \mu$ independent realizations of a uniform random variable with values in $] 0,1]$,

and $i^{2}=-1$. Equation (4) is implemented using the fast Fourier transformation (FFT) algorithm.

2) Generator of non-Gaussian surfaces: For non-Gaussian random fields, the iterative generator developed in [34] is applied in such a way that the generated surface heights have a first order mPDF and a PSD function approximating the target ones. In this algorithm, see Fig. 4, at each iteration $(i)$, a Gaussian surface $\tilde{z}_{\mathrm{G}}^{(i)}$ is generated from the PSD function $s_{Z_{\mathrm{G}}}^{(i)}$ using the Gaussian surface generator described by Eq. (4). The PSD function $s_{Z_{\mathrm{G}}}^{(i)}$ is adapted at each iteration and is equal to the target PSD function, $s_{Z}$, for the first iteration. In order to match the non-Gaussian first order mPDF, using 


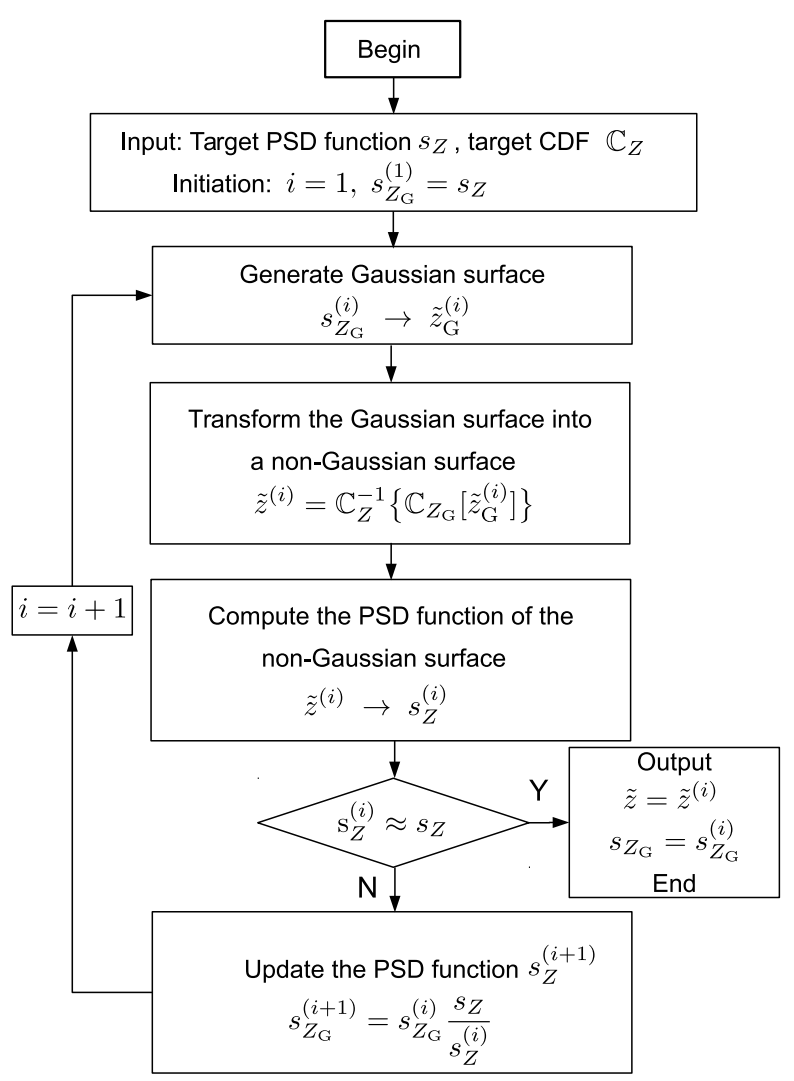

Figure 4. Algorithm to generate non-Gaussian surfaces respecting the target PSD $s_{Z}$, and the target non-Gaussian cumulative distribution function $\mathbb{C}_{Z}$.

the cumulative distribution functions (CDFs), that Gaussian surface is mapped to a non-Gaussian one $\tilde{z}^{(i)}$ following

$$
\tilde{z}^{(i)}(\mathbf{x})=\mathbb{C}_{Z}^{-1}\left\{\mathbb{C}_{Z_{\mathrm{G}}}\left(\tilde{z}_{G}^{(i)}(\mathbf{x})\right)\right\},
$$

where $\mathbb{C}_{Z}$ and $\mathbb{C}_{Z_{\mathrm{G}}}\left(\tilde{z}_{\mathrm{G}}^{(i)}(\mathbf{x})\right)$ are the CDFs of the target nonGaussian distribution evaluated from the first order mPDF $\mathrm{p}_{Z}$ in Sec. II-A and of the Gaussian generated surface $\tilde{z}_{\mathrm{G}}^{(i)}$, respectively. With that mapping, $\tilde{z}$ respects the target PDF, however the PSD function is modified. The PSD function $s_{Z}^{(i)}$ of the non-Gaussian mapped surface is estimated using Fourier analysis. At the end of each iteration, an adapted PSD function is computed following

$$
s_{Z_{\mathrm{G}}}^{(i+1)}=s_{Z_{\mathrm{G}}}^{(i)} \frac{s_{Z}}{s_{Z}^{(i)}},
$$

and is used to generate a new Gaussian surface in the next iteration. The process is repeated until reaching the condition $s_{Z}^{(i)} \approx s_{Z}$.

To perform MCS, a large number of numerical surfaces is required. The first numerical surface is obtained using the iterative algorithm. However, for the next generated surfaces, the converged $s_{Z_{G}}$, see the output box in Fig. 4, is used directly to generate the Gaussian surfaces which are then mapped to obtain the non-Gaussian ones, and the iterative process is thus no longer required.

\section{Estimation of the PSD function}

The PSD function $s_{Z}$, provided that it exists, is the (inverse) Fourier transform of the autocovariance function (ACF). When AFM measurements are available, the PSD function can be evaluated using Fourier analysis [31]. In this paper, because we do not have access to AFM measurements of the surfaces, we use the cutoff self-affine PSD function given by

$$
\frac{s_{Z}\left(\zeta_{r}\right)}{s_{Z}^{0}}= \begin{cases}1 & \text { if } 0 \leq \zeta_{r}<\zeta_{r}^{0} ; \\ \left(\frac{\zeta_{r}}{\zeta_{0}}\right)^{\log _{10}\left(\frac{s_{Z}^{1}}{s_{z}^{0}}\right) / \log _{10}\left(\frac{\zeta_{\max }}{\zeta_{0}}\right)} & \text { if } \zeta_{r}^{0} \leq \zeta_{r} \leq \zeta_{\max } \\ 0 & \text { if } \zeta_{\max }<\zeta_{r} ;\end{cases}
$$

where $\zeta_{r}=\sqrt{\zeta_{1}^{2}+\zeta_{2}^{2}}$, and $s_{Z}^{0}, s_{Z}^{1}, \zeta_{\max }, \zeta_{0}$ are parameters to be identified. That form of PSD function was asymptotically verified for a large range of measured surfaces [40]. The parameters of that PSD function are identified to satisfy the condition that the generated surfaces fit the reported experimental data, i.e. the density of summits, $\bar{N}_{\text {sum }}$, and the mean radius of these summits, $\bar{R}_{\text {sum. }}$. For the cases in which the surface heights follow a Gaussian distribution, from the mean summit radius and the summit density together with the surface height variance, the parameters of that PSD function can be identified using the explicit relations between PSD moments and the summit properties developed by Nayak in [41]. For the non-Gaussian cases considered in this paper, since these relations are not explicitly established, the inverse problem of the parameters identification is solved with an iterative process initialized using Nayak's relations[41].

\section{A MULTI-SCALE STOCHASTIC FRAMEWORK FOR ADHESIVE CONTACT}

To quantify the uncertainty of MEMS structures subjected to stiction, the stochastic model-based multi-scale method developed by the authors in [30] is applied. The generated samples of the contacting surfaces obtained using the generator developed in Sec. II are the input data of the model. Contrarily to the work in [30], non-Gaussian surfaces are considered; apart from that the methodology is similar. The model involves three scales.

- The lower scale is related to the randomness of the contacting surfaces. The characteristic lengths at this scale, $l^{\mathrm{m}}$, are the correlation lengths of the considered surfaces and defined using the condition: $r_{Z}(\boldsymbol{\tau})<0.01 m_{2}$, $\forall\|\boldsymbol{\tau}\|>l^{\mathrm{m}}$

- The mesoscopic scale is related to the adhesive contact behaviors evaluated between two rough surfaces. The characteristic length at this scale, $l^{\text {meso }}$, characterizes the size of these surfaces.

- The upper scale is related to the structural behaviors of the considered micro structures. The characteristic length at this scale, $l^{\mathrm{M}}$, is the size of these structures.

The requirement for the development of the multi-scale model is that $l^{\text {meso }} \ll l^{\mathrm{M}}$. Because $l^{\mathrm{M}}$ is small for MEMS, $l^{\text {meso }}$ and $l^{\mathrm{m}}$ have the same order of magnitude. Therefore, there exist uncertainties in the meso-scale contact behaviors. We should remark that $l^{\text {meso }} \lesssim l^{\text {eff; }}$; therefore, the local property 
of the effective contact zone, see Fig. 1(a), is involved in the multiscale model. The probabilistic behaviors at the upper scale of MEMS structures can thus be evaluated by accounting for these uncertainties.

Toward this end, the straightforward solution is the MCS applied on top of the multi-scale model of the considered structure. The goal of that method is to evaluate $N_{M C}$ statistically independent structural behaviors from which their probability is estimated. To avoid the need of explicit evaluation of a large number of contact forces, a stochastic model for these forces is constructed. The obtained stochastic model-based multi-scale method, illustrated in Fig. 3, consists in:

(i) In the meso-scale model, $m$ surface pairs are generated, and their apparent contact forces, as illustrated in Fig. 2, are evaluated using the semi-analytical contact model developed in [31];

(ii) A stochastic model is constructed based on the $m$ explicitly evaluated forces to efficiently generate the samples of the random apparent contact forces;

(iii) At the structural scale model, we first construct a FE model for the considered structure for which $N_{p}$ samples of the random apparent contact forces are integrated as random contact laws associated to the $N_{p}$ integration points of that model. The MCS is then applied to that model to evaluate $N_{M C}$ statistically independent structural behaviors using $N_{M C}$ sets of $N_{p}$ apparent contact forces generated using the stochastic model.

As the number of explicitly evaluated contact forces for step (ii) is significantly smaller than the number required in step (iii), $m \ll N_{M C} \times N_{p}$, the stochastic model-based multiscale method is more efficient in terms of computational cost than a direct MCS approach. Those three components of the stochastic model-based multi-scale method are summarized in the following.

\section{A. Semi-analytical contact model}

Before describing the semi-analytical contact model, the physical aspects of the adhesive forces involving capillary and $\mathrm{vdW}$ forces are discussed.

\section{1) Adhesive forces:}

Capillary forces: When two hydrophilic surfaces enter into contact in humid air condition they form menisci, which induce an adhesive stress on these surfaces, see illustration in Fig. 1(b). The capillary forces are characterized by two parameters, the water pressure inside the meniscus and the meniscus height. Assuming that the water pressure and the meniscus radius are constant during the interface separation process, the pressure is evaluated at a given relative humidity level $R H$ using Laplace equation as [42], [43]

$$
\Delta P=\frac{\gamma_{\mathrm{LG}}}{r_{K}}=\frac{\mathcal{R} T \ln R H}{V_{m}},
$$

where $\gamma_{\mathrm{LG}}$ is the liquid vapor energy, $V_{m}$ is the liquid molar volume, $\mathcal{R}$ is the universal gas constant, and $T$ is the absolute temperature. In case of water condensation $V_{m}=0.018 \mathrm{~L} / \mathrm{mol}$ and $\gamma_{\mathrm{LG}}=0.072 \mathrm{~N} / \mathrm{m}$ at $T=300 \mathrm{~K}$. The MEMS contact surfaces considered are nominally flat, thus when the contacting surfaces are perfectly hydrophilic, the meniscus height is approximated as $h_{C}=-2 r_{K}$, where $r_{K}$ is the Kelvin radius given by [43]

$$
r_{K}=\frac{\gamma_{\mathrm{LG}} V_{m}}{\mathcal{R} T \ln R H}<0
$$

Van der Waals force: Based on the Lennard-Jones potential, the expression of vdW adhesion energy per unit area, $\omega_{\mathrm{vdW}}$, is deduced from the work expended to move the two half spaces undergoing vdW interactions from the equilibrium distance to infinity [17], [44], [45] leading to

$$
\omega_{\mathrm{vdW}}=\frac{\mathfrak{H}}{16 \pi D_{0}^{2}}
$$

where $\mathfrak{H}$ is the Hamaker constant, and $D_{0}$ is the equilibrium distance at which the force between the two half spaces is zero and is given by $D_{0}=(2 / 15)^{1 / 6} r_{0}$ with $r_{0}$ the finite distance at which the inter-molecular potential is zero. The parameters of $\mathrm{vdW}$ stress for polysilicon-the considered material in this paper-are: the Hamaker constant $\mathfrak{H}=18.65 \times 10^{-20} \mathrm{~J}$ through dry air [46], and $\mathfrak{H}=9.75 \times 10^{-20} \mathrm{~J}$ through water [46] which can be due to the menisci; the distance $r_{0}=2.09 \AA$ leading to $D_{0}=1.49 \AA$. The vdW interaction is modeled using Dugdale assumption, i.e. the stress is constant inside the contact range $\left[0, h_{\mathrm{vdW}}\right]$ and vanishes outside this range. For silicon, $h_{\mathrm{vdW}}=0.97 \times 2^{1 / 6} r_{0}=2.28 \AA$ [45]. The stress of the vdW interaction $\sigma_{\mathrm{vdW}}$ is evaluated from the relationship $\omega_{\mathrm{vdW}}=-\sigma_{\mathrm{vdW}} \times h_{\mathrm{vdW}}$.

2) Semi-analytical adhesive contact model: The contact between two rough surfaces, e.g. $z_{1}$ versus $z_{2}$, can be modeled as the contact between an equivalent surface $z=z_{1}+z_{2}$ and a flat surface [11]. The semi-analytical adhesive contact model developed in [31] is thus applied on this equivalent problem. The model is summarized in the following, firstly for the case of capillary adhesive forces, and then for the case both capillary and vdW forces are considered.

Because the Laplace pressure is weak $(<400 \mathrm{MPa})$ in comparison with the stiffness of polysilicon, at the equilibrium state for which the adhesive force and elastic repulsive force are equal, the total condensing water area is much bigger than the physical contact area. Based on this separation, the model accounting for capillary forces was developed in two steps, see [31] for details:

(i) Evaluation of the elastic repulsive forces by identifying the physical contacting asperities from the surface topology, fitting these contacting asperities profiles by spheres, and then applying Hertz contact model [14] to evaluate the elastic repulsive contact forces and the physical contact areas for each asperity; and

(ii) Evaluation of the adhesive force by evaluating the area of condensing water using the water height $h_{C}$, and the surface topology, and then multiplying that area with the Laplace pressure, Eq. (8).

The apparent adhesive contact force is obtained by adding these forces and then dividing the result by the apparent area of generated surfaces. With this contact model, the saturation effect in which the menisci are merged to each other [6], is accounted for [31]. As illustrated in Fig. 2, the apparent force versus distance curve has two parts: the adhesive part in which forces are negative resulting from the dominant adhesive 
interaction and the repulsive part in which forces are positive resulting from the dominant elastic interaction.

In order to account for the $\mathrm{vdW}$ phenomenon, due to its sub-nanometer range, its effects can be evaluated when performing step (i). In that case, the elastic repulsive forces and the adhesive forces resulting from the vdW phenomenon are evaluated by applying the Maugis model [17] instead of the Hertz model [30].

Since the semi-analytical contact model does not account for irreversible behaviors, we have assessed in Appendix A that these phenomena can be neglected for the cases studied in Sec. IV. To this end, the ratio between the contact area undergoing irreversible deformation and the total physical contact area is estimated and is shown to remain negligible. Note that in Eq. (8) we have assumed a constant Laplace pressure, which is relevant in the case of release stiction study, but not during cyclic loading, see the discussion in [29], [47], and is thus consistent with neglecting irreversible behaviors induced by cyclic loading.

\section{B. Construction of the stochastic model of the random appar- ent adhesive contact forces}

A parametrization process is applied to identify the parameters vector representing the apparent adhesive contact forces. Because the apparent adhesive contact forces evaluated on the different generated surfaces exhibit a scatter as illustrated in Fig. 2, the parameters vector is considered as a random variable. The stochastic model is then constructed to generate the samples of apparent contact forces through that random vector.

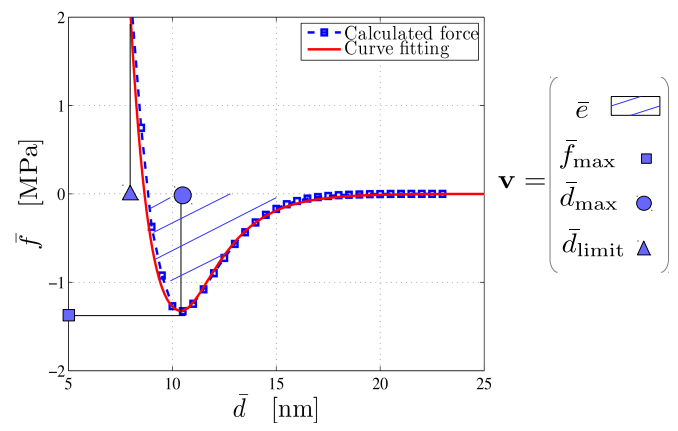

Figure 5. The components of the parameters vector $\mathbf{v}$, and the curve fitting of a typical apparent adhesive contact force using the analytical function, Eq. (12), whose parameters are evaluated from $\mathbf{v}$.

1) Parametrization of the apparent adhesive contact forces: An analytical function used to fit the apparent adhesive contact forces is derived from the Morse potential with the coefficients evaluated from the characterized parameters. Let $\bar{f}(\bar{d})$, with $\bar{d}$ the contact distance, be the apparent adhesive contact forces evaluated using the semi-analytical contact model described in Sec. III-A. These forces are characterized by 4 parameters, illustrated in Fig. 5, including (i) the maximum pull-out adhesive force $\bar{f}_{\max }(>0)$, (ii) the distance $\bar{d}_{\max }$ at which $\bar{f}$ reaches $-\bar{f}_{\text {max }}$, (iii) the meso-scale apparent adhesion energy $\bar{e}(>0)$, and (iv) the threshold distance $\bar{d}_{\text {limit }}$ at which $\bar{f}$ reaches $\bar{f}_{\text {limit }}(>0)$, the maximum considered compressive force of interest, which can be chosen as the maximum of the $\bar{f}_{\text {max }}$ evaluated for different generated surfaces in the MCS. Assembling these parameters, we obtain the parameters vector $\mathbf{v}$ as

$$
\mathbf{v}=\left[\bar{e}, \bar{f}_{\max }, \bar{d}_{\max }, \bar{d}_{\text {limit }}\right]^{\mathrm{T}} .
$$

The following function, derived from the Morse potential, is used to fit the apparent adhesive contact forces

$\phi(\bar{d})= \begin{cases}\bar{f}_{\max }\left(\mathrm{e}^{-2 a_{\text {right }}\left(\bar{d}-\bar{d}_{\text {max }}\right)}-2 \mathrm{e}^{-a_{\text {right }}\left(\bar{d}-\bar{d}_{\max }\right)}\right) & \text { if } \bar{d} \geq \bar{d}_{\text {max }} \\ \bar{f}_{\max }\left(\mathrm{e}^{-2 a_{\text {left }}\left(\bar{d}-\bar{d}_{\max }\right)}-2 \mathrm{e}^{-a_{\text {left }}\left(\bar{d}-\bar{d}_{\max }\right)}\right) & \text { if } \bar{d}<\bar{d}_{\text {max }}\end{cases}$

Because that analytical function has 4 fitting coefficients, $\left\{\bar{f}_{\text {max }}, \bar{d}_{\text {max }}, a_{\text {right }}, a_{\text {left }}\right\}$, it is consistent with the dimension of the parameters vector defined in Eq. (11) and can be readily obtained. As it is observed from Fig. 5, the proposed analytical function described in Eq. (12) approximates well the evaluated adhesive contact forces. Therefore, the parameters vector $\mathbf{v}$, defined by Eq. (11), is representative of the apparent adhesive contact forces.

2) Stochastic model of random apparent adhesive contact forces: Considering the parameters vectors $\mathbf{v}$ as a random vector denoted by $\mathbf{V}$, a stochastic model is constructed to generate realizations of $\mathbf{V}$. The input data for the construction process consist of $m$ explicitly evaluated apparent contact forces, from which the set of $m$ corresponding physical parameters vectors $\left\{\mathbf{v}^{(1)}, \ldots, \mathbf{v}^{(m)}\right\}$-samples of $\mathbf{V}$-is obtained using the parametrization process described in Sec. III-B1. That stochastic model [30], summarized in Appendix B, verifies the condition of approximation in terms of distribution, i.e. the distribution of generated samples approximates the one of the input data as shown in Appendix B.

\section{Upper scale FE model for cantilever beam structures}

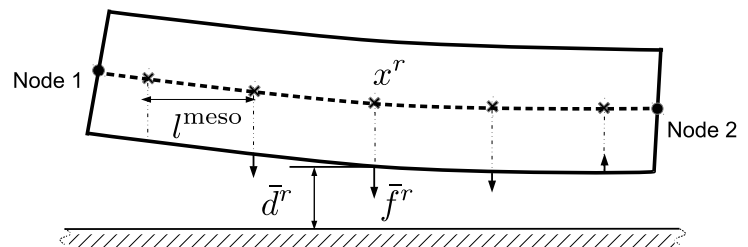

Substrate

Figure 6. 1D beam element. Each integration point is associated with a different sample of the random apparent adhesive contact forces, $\bar{f}^{r}$, whose evaluated values depend on the contact distance $\bar{d}^{r}$, see Fig. 2.

Because the cantilever beam is one of the most ubiquitous structures in the context of MEMS, and particularly was used for stiction tests, it is considered in this paper. In the following, we develop the FE model for beams subjected to adhesive contact, and we integrate that model into a MCS to get the probability of the beam stiction behaviors.

Construction of the FE model: The structural scale model is a 1D FE model, implemented using Euler-Bernoulli beam theory [48] and discretized into $N_{e}$ elements of uniform length $l_{e}$. To evaluate the nodal adhesive contact forces, the rectangle quadrature rule is applied, i.e. the integration points are equally spaced with the distance $l_{\text {quad }}$ which is 
enforced to be $l^{\text {meso }}$ in this work, see Fig. 6 for the beam element case. With this enforcement, the size effect at the mesoscopic scale is always accounted for at the upper scale when performing the convergence analysis, i.e. by gradually reducing the meso-scale length $l^{\text {meso }}$, see Appendix $\mathrm{C}$ for the case studied in Sec. IV. The total number of integration points is $N_{p}$. The interaction between the beam bottom surface and the substrate is modeled by associating $N_{p}$ apparent adhesive contact forces, $\bar{f}^{r}$ with $r=1, \cdots, N_{p}$, generated by using the stochastic model constructed in Sec. III-B2. This modeling method requires that the correlation of the contact forces of neighboring integration points is negligible.

Because the nodal contact forces depend on the displacement of the beam, the problem is non-linear. The FE model is hence solved using the Newton-Raphson method -an iterative algorithm - to reach equilibrium between internal forces resulting from the beam deformation, and external forces resulting from the adhesion contact and the applied forces. Details on the FE formulation and implementation can be found in [30].

Remark on the consistency of the generated surface size. In order to ensure the consistency with the evaluation of the nodal contact forces, the size of the generated surfaces used to evaluate the apparent contact forces in Sec. III-A is chosen as $l^{\text {meso }} \times w$, see Appendix $\mathrm{C}$ for the identification of $l^{\text {meso }}$ with the element length $l_{e}$ applied for the case studied in Sec. IV. Furthermore, because the distance between two integration points is larger than the correlation length $l_{\text {quad }}>l^{\mathrm{m}}$, the correlation of the neighboring contact forces is negligible [30].

Stiction simulation: To simulate the stiction, a loadingunloading process is implemented [30], [45]. In this process, an increasing artificial force is applied to put the cantilever beam into contact with the substrate. After the beam reaches a certain deformation energy higher than the expected value of the adhesion energy, the applied force is released progressively. At the end of the process, the cantilever beam could reach the stiction failure, see Fig 1(a), with either S-shape configuration or arc-shape configuration.

\section{CASE STUdy}

In this section, the developed method is applied to model the stiction experiments reported in [5]. In this experimental campaign, arrays of micro polysilicon cantilever beams were fabricated, and stiction tests were performed through the three following steps. First, the cantilever beams were put on the substrates made of silicon or polysilicon. Then, the clamped ends of the beams were moved up to reach a given height $h$. Due to the adhesive stress resulting from vdW and capillary interactions, the beams exhibit the S-shape stiction configuration, see Fig. 1(a). The crack length, $l_{s}$, was measured and used to evaluate the upper scale apparent adhesion energy $\Gamma\left(\right.$ in $\left.\mathrm{J} / \mathrm{m}^{2}\right)$ as

$$
\Gamma=\frac{3}{2} E \frac{h^{2} t^{3}}{l_{s}^{4}},
$$

where $E$ is the Young's modulus of the beam, and $t$ is the beam thickness [49]. For polysilicon, $E=164 \mathrm{GPa}$.

Three sets of tests were conducted with two different beam sets B1, B2 and two different substrates Sub1, Sub2: (i) pair a: between beam set B1 and substrate Sub1, (ii) pair b: between beam set B2 and substrate Sub1, and (iii) pair c: between beam set B1 and substrate Sub2. Note that all the contact surfaces were treated to become super-hydrophilic, i.e. characterized by an almost zero contact angle. The height distribution moments of the involved contacting surfaces, including the bottom surfaces of two different sets of cantilever beams, B1, B2, and the two substrate surfaces Sub1, Sub2, are reported in Tab. I. In Tab. I, two spatial properties, the mean radius of summits and the density of summits, are also reported. The values of the mean summit radius and summit density depend on the sampling distance. It was reported to be $20 \mathrm{~nm}$ in reference [50] which was used for the numerical topology processing of the considered tests in [5].

The geometrical dimensions of the beams were given as: the beam thickness $t=2.6 \mu \mathrm{m}$, the beam length $L=1500 \mu \mathrm{m}$, and the beam width $w=30 \mu \mathrm{m}$. The gap is $h=20 \mu \mathrm{m}$ for pairs a and b. For pair c, the value $h$ was not reported in [5]. Because the reported values of apparent adhesion energies for pair c are much smaller in comparison with pairs a, b, we chose $h=2 \mu \mathrm{m}$ in order to ensure that the modeled beams reach the S-shape configuration. Due to the fabrication and conducted experiment processes, the geometrical dimensions can also vary. The effect of the geometrical dimensions uncertainties should thus be considered and compared with the one resulting from the random surfaces when predicting the probability of the adhesion energies.

In the following the method developed in Secs. II and III is implemented to model that experiment. An extension of the model to account for the randomness of the geometrical dimensions is then realized.

\section{A. Characterization and simulation of contacting surfaces}

Estimation of the first order $m P D F$ : Based on the reported experimental data including roughness, skewness, and kurtosis, see Tab. I, the non-Gaussian PDFs of surface heights are estimated using the ME method developed in Sec. II-A. The estimated PDFs are illustrated and compared with the Gaussian ones in Fig. 7. In all cases, the values of skewness are negative. As a result, in comparison with the distributions obtained using the ME method, the Gaussian distributions for which the skewness is zero are more shifted to the higher asperities. Consequently, the apparent adhesion energy can be underestimated if one uses a Gaussian distribution to model the surface heights. This fact will be quantified by a sensitivity analysis and will be confirmed by numerical results in Sec. V.

Estimation of the PSD function: The parameters of the cutoff self-affine PSD function, defined in Eq. (7), are identified following the method described in Sec. II-C to satisfy the reported spatial parameters including the density of summits and the mean radius of theses summits, reported in Tab. I. The identified parameters for the considered experimental surfaces are reported in Tab. II.

Generation of surfaces: Based on the estimated first order mPDFs and the constructed PSD functions, the numerical surfaces are generated using the method described 
Table I

COMPARISON BETWEEN AFM MEASUREMENT SURFACES REPORTED IN [5] AND SIMULATION SURFACES, GENERATED USING GAUSSIAN DISTRIBUTION (SIM-G) AND NON-GAUSSIAN DISTRIBUTION (SIM-NG) OF SURFACE HEIGHTS, IN TERMS OF ROUGHNESS, SKEWNESS, KURTOSIS, MEAN RADIUS OF SUMMITS $\left(\bar{R}_{\mathrm{SUM}}\right)$, AND DENSITY OF SUMMITS $\left(\bar{N}_{\mathrm{SUM}}\right)$.

\begin{tabular}{|c|c|c|c|c|c|c|c|c|c|c|c|c|}
\hline & \multirow{2}{*}{\multicolumn{6}{|c|}{$\begin{array}{c}\text { Bottom surfaces } \\
\text { of the cantilever beams (polysilicon) }\end{array}$}} & \multicolumn{6}{|c|}{ Substrates } \\
\hline & & & & & & & & & & & & \\
\hline & \multicolumn{3}{|c|}{ B1 } & \multicolumn{3}{|c|}{ B2 } & \multicolumn{3}{|c|}{ Sub1 (silicon wafer) } & \multicolumn{3}{|c|}{ Sub2 (polysilicon) } \\
\hline & AFM & Sim-NG & Sim-G & AFM & Sim-NG & Sim-G & AFM & Sim-NG & Sim-G & AFM & Sim-NG & Sim-G \\
\hline$r m s[\mathrm{~nm}]$ & 1.7 & 1.7 & 1.7 & 3.4 & 3.4 & 3.4 & 0.17 & 0.17 & 0.17 & 5.5 & 5.5 & 5.5 \\
\hline$\gamma[-]$ & -0.78 & -0.78 & 0 & -0.76 & -0.76 & 0 & -0.31 & -0.31 & 0 & -1.1 & -1.1 & 0 \\
\hline$\beta[-]$ & 4.6 & 4.6 & 3 & 3.9 & 3.9 & 3 & 3.1 & 3.1 & 3 & 5.1 & 5.1 & 3 \\
\hline $\bar{R}_{\text {sum }[\mu \mathrm{m}]}$ & 0.41 & 0.41 & 0.30 & 0.84 & 0.85 & 0.56 & 3.0 & 3.0 & 3.0 & 0.12 & 0.14 & 0.09 \\
\hline $\bar{N}_{\text {sum }}\left[\mu \mathrm{m}^{-2}\right]$ & 68 & 63 & 71 & 20.9 & 20.0 & 29.2 & 111.5 & 109 & 115 & 48 & 59 & 68 \\
\hline
\end{tabular}

pair a: B1 vs Sub1, pair b: B2 vs Sub1, pair c: B1 vs Sub2

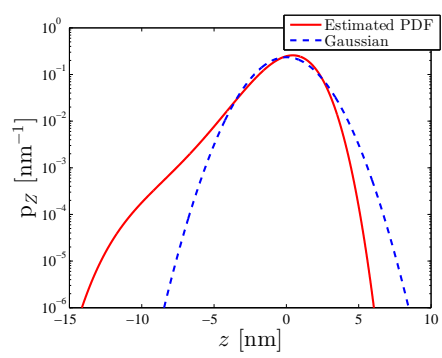

(a)

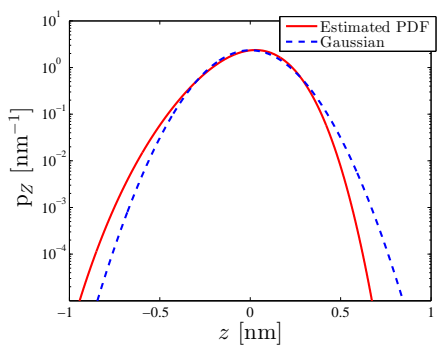

(c)

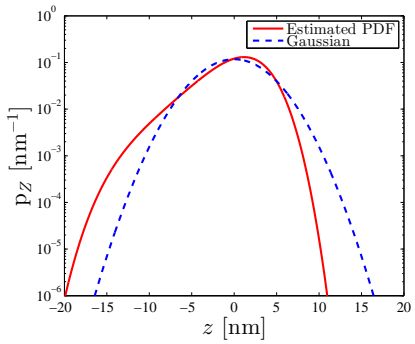

(b)

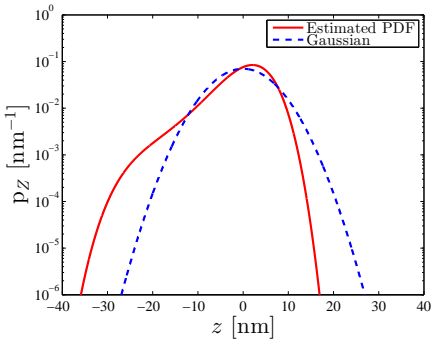

(d)

Figure 7. The estimated first order mPDFs using ME method with the moments given in Tab. I: : B1 (a), B2 (b), Sub1 (c), Sub2 (d).

Table II

THE PARAMETERS OF THE CONSTRUCTED PSD FUNCTIONS GIVEN BY EQ. (7).

\begin{tabular}{lcccc} 
& B1 & B2 & Sub1 & Sub2 \\
\hline$s_{Z}^{0}\left[\mathrm{~nm}^{4}\right]$ & 525 & 1438 & 3.5 & 2471 \\
$s_{Z}^{1}\left[\mathrm{~nm}^{4}\right]$ & 0.8 & 0.3 & 0.001 & 0.2 \\
$\zeta_{0}\left[\mathrm{rad} \cdot \mu \mathrm{m}^{-1}\right]$ & 32 & 12 & 10 & 56 \\
$\zeta_{\max }\left[\mathrm{rad} \cdot \mu \mathrm{m}^{-1}\right]$ & 138 & 125 & 264 & 157
\end{tabular}

in Sec. II-B. The generated surfaces are illustrated in Fig. 8 for 2D illustration and in Fig. 9 for 1D illustration. As it is observed in Fig. 10, the PSD function of the generated nonGaussian surfaces preserves well the target one confirming the convergence of the iterative algorithm described in Sec. II-B.

From the generated surfaces, the summits can be identified togethe with their radius and their heights. The joint distributions of the summit heights and summit radii (logarithmic scaled) obtained for two cases -Gaussian distribution and non-Gaussian distribution of surface heights, see Fig. 7- are illustrated in Fig. 11 for the surface B2. As it is observed from Fig. 11, by accounting for the non-Gaussian properties, the heights of summits involved in the physical interaction, i.e. the highest ones, are less scattered, and the summits radii are larger, i.e. the mean of summits radii is $0.85 \mu \mathrm{m}$ for nonGaussian case and $0.56 \mu \mathrm{m}$ for Gaussian case. This fact is also observed in Fig. 9. When the height distribution of the highest summits is less scattered, more summits are simultaneously involved in the contact interaction, and consequently, the adhesion forces increase while their uncertainties are reduced following the law of large numbers. For example in [6], using an analytical model, authors have shown that the adhesion energies are larger for larger summit radii and uniformly distributed summit heights.

The comparison between measurement data and simulated surfaces is reported Tab. I. The moments, including roughness, skewness and kurtosis, are modeled with accuracy. For the spatial properties, because the PSD functions are indirectly estimated using the cutoff self-affine formulation, Eq. (7), there exist errors in the density and the mean radius of the summits. These errors are negligible for B2 and Sub1, 


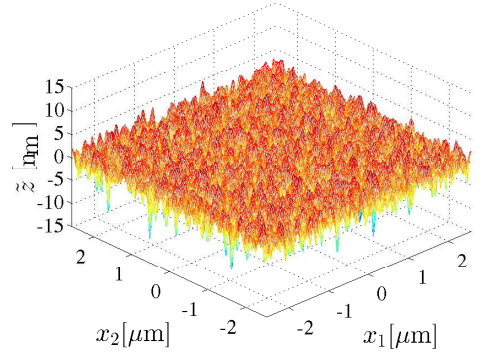

(a)

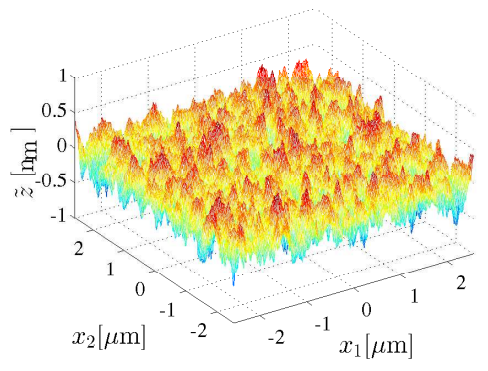

(c)

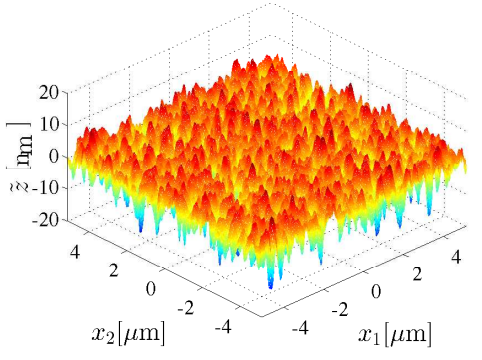

(b)

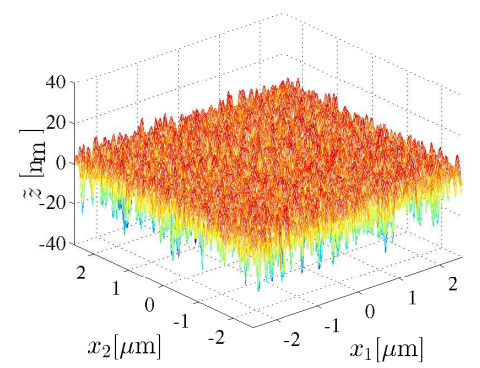

(d)

Figure 8. Examples of the generated non-Gaussian surfaces: B1 (a), B2 (b), Sub1 (c), Sub2 (d).

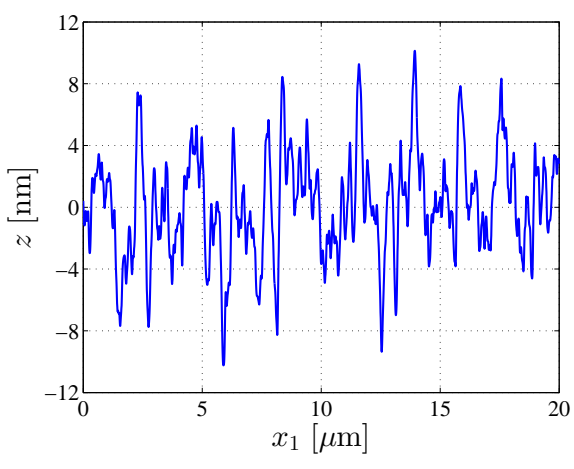

(a)

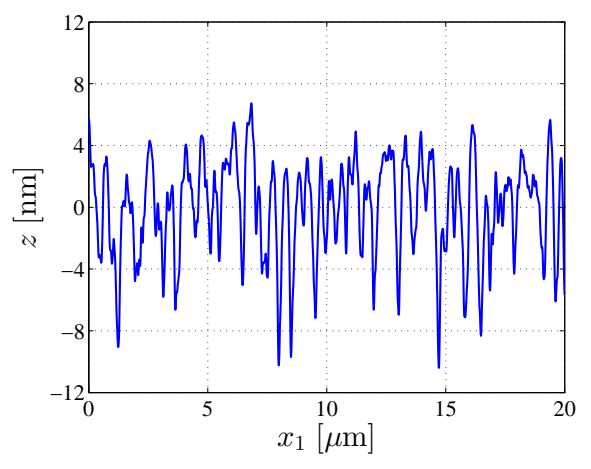

(b)

Figure 9. Comparison of generated surfaces between two cases: (a) using the Gaussian distribution of surface heights, and (b) using the non-Gaussian distribution of surface heights. The surface B2 is considered.

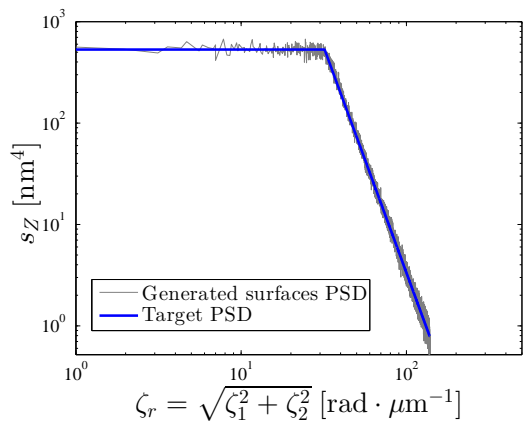

Figure 10. Comparison of the PSD functions for the case of B1 between the target one constructed using Eq. (7) with the coefficients given in Tab. II, and the one evaluated from their corresponding non-Gaussian generated surfaces. moderate for B1, and significant for Sub2, i.e. $17 \%$ for the mean radius of summits and $23 \%$ for the density of summits. As a result, the numerical results might deviate from the experimental data for the pair c in which the substrate Sub2 is involved. This fact will be confirmed during the comparison between numerical results and experimental ones in Sec. V.

Remarks. Among the cases, the distribution of surface heights in case of Sub1 is close to a Gaussian distribution see Tab. I. As a result, the errors of the generated Gaussian surfaces compared to the AFM measurements in terms of the mean radius and the density of the summits are small and comparable with the generated non-Gaussian surfaces. However, in the other cases, the errors are significant between Gaussian generated surfaces and AFM measurements. In fact, the generated Gaussian surfaces can be improved by constructing the PSD function toward the goal of matching the mean radius and the density of summits for the Gaussian 


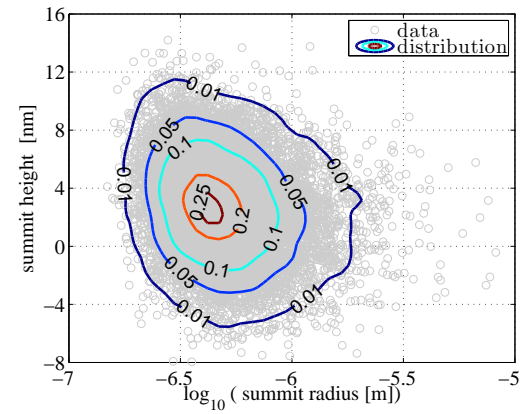

(a)

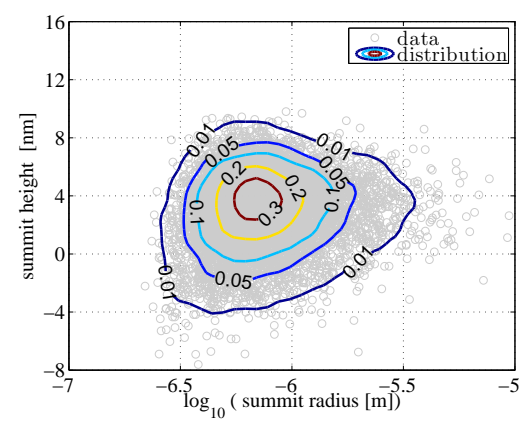

(b)

Figure 11. The joint distributions of the summit heights and the logarithmic scaled summit radii evaluated from the generated surfaces for two cases of surface heights distributions: (a) Gaussian and (b) non-Gaussian. The surface B2 is considered.

generated surfaces as performed in [30]. Here, since the surface height distributions of the considered cases including B1, B2, and Sub2 expose a strong non-Gaussian characters, the non-Gaussianity is required to be accounted for to further improve the accuracy when representing the real surfaces using the generated ones.

\section{B. Propagation of surface randomness using the stochastic model-based multi-scale method}

The stochastic model-based multi-scale method, discussed in Sec. III and illustrated in Fig. 3, is implemented for the study case.

Evaluation of the apparent adhesive contact forces: Using the surfaces generator developed in Sec. IV-A, $m=5000$ couples of surfaces of size $l^{\text {meso }} \times w$ are generated. The value of $l^{\text {meso }}$ is chosen as $1.5 \mu \mathrm{m}$ following the argumentation given in Appendix C. The semi-analytical contact model described in Sec. III-A is applied to evaluate the apparent contact forces, with some realizations illustrated in Fig. 12(b, e, h). Also in Fig. 12(a, d, g), the apparent contact forces obtained using a Gaussian distribution of surface height are illustrated. As it is observed from the comparison between the first two columns of Fig. 12, the adhesive forces are underestimated when considering Gaussian distributions as expected. We note that irreversible behaviors are negligible for the considered cases, see the detailed justification in Appendix A.

Sensitivity analysis: A sensitivity analysis is implemented to study the dependency of the meso-scale apparent contact forces on the statistical moments of the contact surfaces, e.g. standard derivation, skewness, and kurtosis. To this end, the normalized derivatives of the mean and of the standard deviation of the meso-scale apparent adhesion energy $\bar{e}$, with respect to the normalized statistical moments of the contact surfaces, are evaluated for the case of pair b are illustrated in Fig. 13. It is observed from Fig. 13 that both the mean and standard deviation of the meso-scale apparent adhesion energy strongly depend on the skewness and kurtosis. On the one hand, an increase of the kurtosis or of the standard deviation of the asperity heights increases the number of asperities in the tails, and thus decreases the average meso-scale apparent energy. On the other hand, an increase of the skewness leads to a less uniform distribution of higher asperities as illustrated in Fig. 9, and thus to a decrease of the average meso-scale apparent energy. In addition, these sensitivities significantly increase, in absolute value, when reducing the humidity level. This confirms the importance of skewness and kurtosis when predicting adhesive contact.

Construction of the stochastic model: The evaluated apparent contact forces are the input data to construct the stochastic models described in Sec. III-B, see details of their construction in Appendix B-C. The apparent contact forces generated using the stochastic model, as illustrated in Fig. 12(c, f, i), represent the randomness observed in the explicitly evaluated apparent contact forces illustrated in Fig. 12(b, e, h).

Evaluation of the probabilistic behavior of micro cantilever beams subjected to stiction phenomenon: Using the constructed stochastic model, $N_{p}=l / l_{\text {meso }}=1000$ apparent contact forces are generated efficiently. Integrating these $N_{p}$ apparent contact forces as random contact laws into the FE model of the cantilever beam described in Sec. III-C, and using that model to simulate the stiction process, a corresponding $\mathrm{S}$ shape stiction failure is obtained. From the obtained shape, the crack length is measured and the corresponding upper scale apparent adhesion energy is evaluated using Eq. (13). Applying the MCS method, that process is independently repeated $N_{M C}=1000$ times. In Fig. 14(a), the obtained Sshape configurations for the case of pair $\mathrm{b}$ at $R H=65 \%$ are visualized, and in Fig. 14(b), the histogram of the corresponding crack lengths is illustrated. The simulations result in a set of $N_{M C}$ values of the apparent adhesion energies from which their distribution is evaluated. The results of the adhesive contact energies for the three tests at different humidity levels, for the cases of Gaussian and non-Gaussian contact surfaces, are illustrated and compared with the experimental data in Fig. 15(a, c, e).

\section{Accounting for the geometrical uncertainties}

In order to account for the effect of the randomness of the geometrical dimensions, including beam width, beam length, beam thickness, and the gap height, on the apparent adhesion energies, the current model is extended by considering these dimensions as random variables. For a sake of study purposes, these parameters are modeled as Gaussian random variables with the standard deviation equal to $2.5 \%$ of their mean values. 


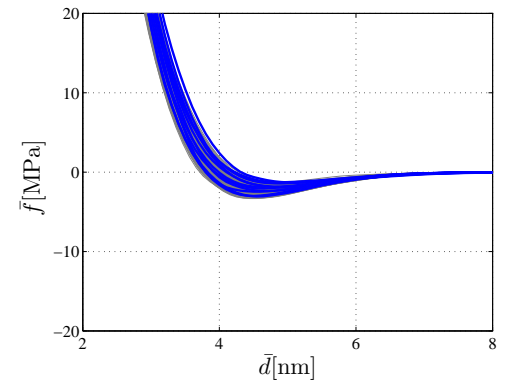

(a)

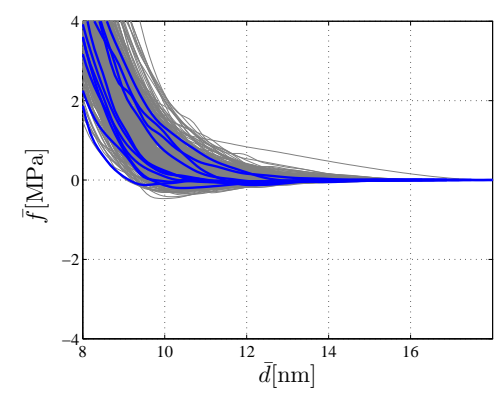

(d)

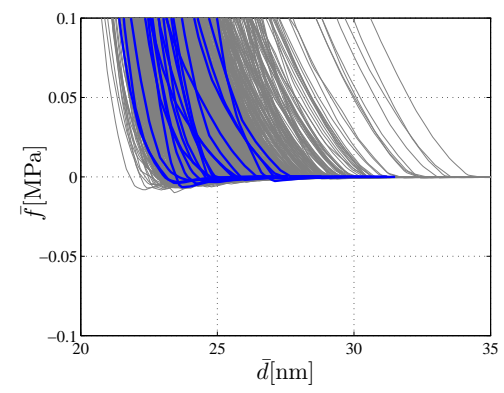

(g)

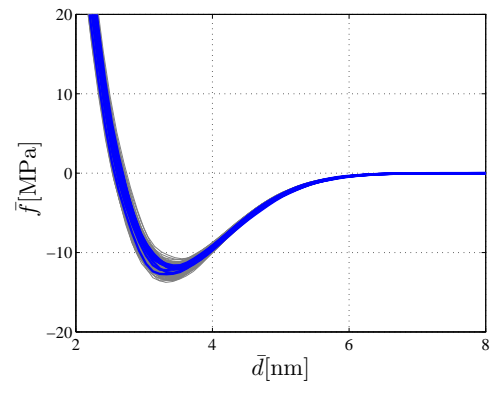

(b)

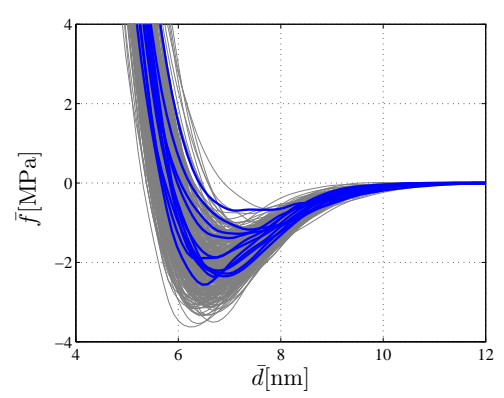

(e)

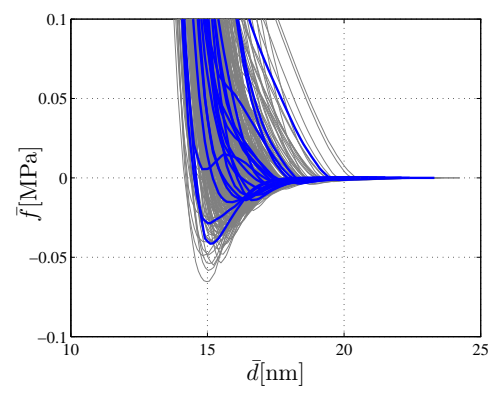

(h)

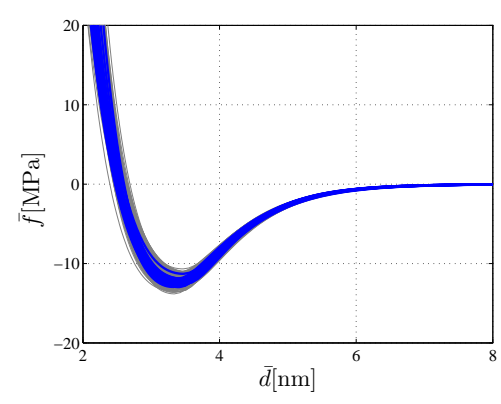

(c)

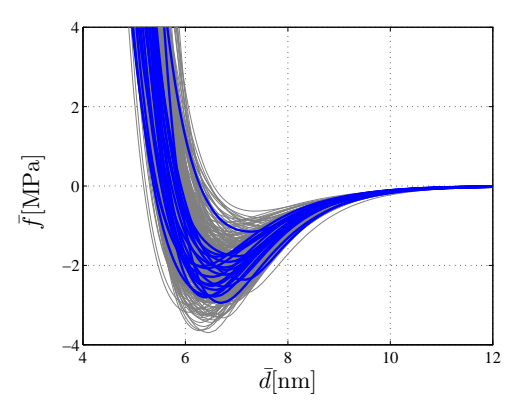

(f)

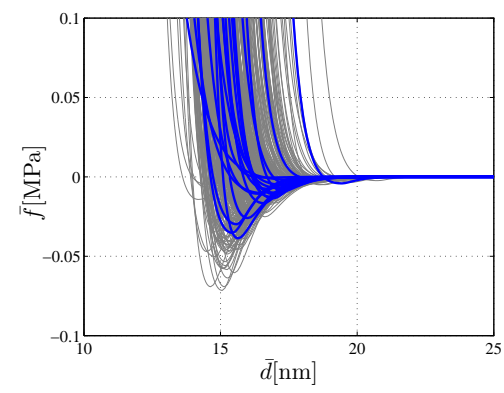

(i)

Figure 12. The apparent contact forces: first column, ( $a, d, g)$, evaluated forces for Gaussian generated surfaces corresponding to the pair a, the pair b, and the pair c respectively; second column, (b, e, h) evaluated forces for non-Gaussian generated surfaces corresponding to the pair a, the pair b, and the pair $\mathrm{c}$ respectively; third column, (c, f, i), generated forces using the stochastic model developed in Appendix B-C corresponding to the pair a, the pair b, and the pair c respectively. For each figure, 20 realizations are highlighted out of the 200 ones. Humidity condition: $R H=0.65$.

The obtained results are compared with the experimental data in Fig. 15(b, d, f).

\section{Discussion}

In the following, we bring out the comparison between the experimental results and the numerical results evaluated for three cases: (G) Gaussian contacting surfaces, (NG) nonGaussian contacting surfaces, and (GEO) extending the case (NG) to account for the geometrical uncertainties. The comparison is conducted with the apparent adhesion energies illustrated in Fig. 15, and the crack lengths obtained from the numerical model and from the experiments as reported in Tabs. III. Based on that comparison, we discuss four issues: the effect of non-Gaussian height distribution of the contact surfaces, the evolution of the adhesion energies and of their uncertainty with the humidity level and the roughness, the effect of the uncertain geometrical dimensions, and the validation of the developed method.
Effect of the non-Gaussian distribution of contact surface heights: As it is observed from the first column of Fig. 15 when accounting for the non-Gaussian effect, the numerical results are significantly more accurate in predicting the experimental results. For these considered cases, the apparent adhesion energies are increased and their uncertainties are decreased when accounting for non-Gaussian properties. It is explained by the fact that the heights of contacting asperities are less prone to scatter, see Fig. 9. Conversely, if the asperities heights had been prone to more scatter, e.g. if the skewness of surface heights had been positive, the apparent adhesion energy would have decreased as compared with the Gaussian cases. The effect can be quantified from Fig. 13, e.g. at $R H=0.44$, a $10 \%$ augmentation in terms of kurtosis or skewness leads to a reduction respectively of $89 \%$ and $64 \%$ in terms of the mean of the meso-scale apparent adhesion energies. 
Table III

THE COMPARISON OF THE CRACK LENGTHS $l_{s}$ IN [ $\mu \mathrm{M}$ ] OBTAINED FROM EXPERIMENTS [5] AND FROM NUMERICAL MODELS FOR PAIRS A, B, C AT DIFFERENT HUMIDITY LEVELS. TWO NUMERICAL MODELS ARE REPORTED: MODEL NG FOR WHICH NON-GAUSSIAN SURFACES ARE CONSIDERED, AND MODEL GEO WHICH EXTENDS THE NG MODEL WITH THE RANDOM GEOMETRICAL DIMENSIONS. THE NUMERICAL RESULTS ARE REPORTED UNDER THE FORM: MEAN \pm STANDARD DEVIATION. THE VALUES OF EXPERIMENTAL RESULTS ARE OBTAINED FROM THE ILLUSTRATION OF APPARENT ADHESION ENERGIES REPORTED IN [5].

\begin{tabular}{l|c|c|c|c|c|c}
\multicolumn{8}{c}{ Pair a } \\
\hline Humidity levels & 0.45 & 0.6 & 0.7 & 0.75 & 0.8 & 0.9 \\
\hline$l_{s}$ (experiment) & $718 \pm 28$ & $604 \pm 24$ & $582 \pm 24$ & $503 \pm 20$ & $465 \pm 15$ & $362 \pm 18$ \\
& & & & & & \\
$l_{s}$ (model NG) & $716 \pm 9$ & $569 \pm 6$ & $494 \pm 4$ & $448 \pm 3$ & $420 \pm 3$ & $353 \pm 3$ \\
$l_{s}$ (model GEO) & $715 \pm 20$ & $567 \pm 14$ & $494 \pm 12$ & $448 \pm 11$ & $420 \pm 10$ & $353 \pm 8$ \\
\hline Bias error & $0.4 \%$ & $6 \%$ & $15 \%$ & $11 \%$ & $10 \%$ & $3 \%$ \\
\hline
\end{tabular}

\begin{tabular}{l|c|c|c|c|c|c}
\multicolumn{7}{c}{ Pair b } \\
\hline Humidity levels & 0.44 & 0.55 & 0.67 & 0.8 & 0.88 & 0.92 \\
\hline$l_{s}$ (experiment) & $952 \pm 60$ & $857 \pm 31$ & $705 \pm 24$ & $564 \pm 22$ & $519 \pm 17$ & $420 \pm 10$ \\
& & & & & & \\
$l_{s}$ (model NG) & $1031 \pm 38$ & $878 \pm 26$ & $710 \pm 16$ & $538 \pm 8$ & $434 \pm 4$ & $391 \pm 3$ \\
$l_{s}$ (model GEO) & $1031 \pm 45$ & $878 \pm 34$ & $710 \pm 23$ & $537 \pm 14$ & $434 \pm 10$ & $390 \pm 9$ \\
\hline Bias error & $8 \%$ & $3 \%$ & $0.7 \%$ & $5 \%$ & $16 \%$ & $7 \%$ \\
\hline
\end{tabular}

\begin{tabular}{l|c|c|c|c|c|c}
\multicolumn{7}{c}{ Pair c } \\
\hline Humidity levels & 0.6 & 0.65 & 0.72 & 0.8 & 0.85 & 0.9 \\
\hline$l_{s}$ (experiment) & $770 \pm 62$ & $767 \pm 60$ & $593 \pm 44$ & $438 \pm 21$ & $383 \pm 12$ & $289 \pm 12$ \\
& & & & & & \\
$l_{s}$ (model NG) & $1200 \pm 131$ & $1014 \pm 99$ & $752 \pm 61$ & $436 \pm 20$ & $301 \pm 9$ & $192 \pm 3$ \\
$l_{s}$ (model GEO) & $1201 \pm 131$ & $1016 \pm 105$ & $752 \pm 68$ & $436 \pm 24$ & $303 \pm 11$ & $192 \pm 5$ \\
\hline Bias error & $56 \%$ & $32 \%$ & $27 \%$ & $0.5 \%$ & $21 \%$ & $34 \%$ \\
\hline
\end{tabular}

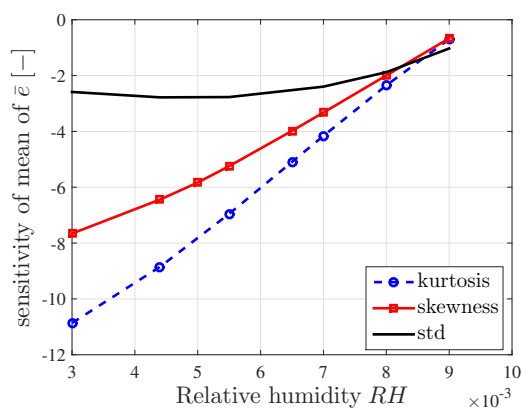

(a)

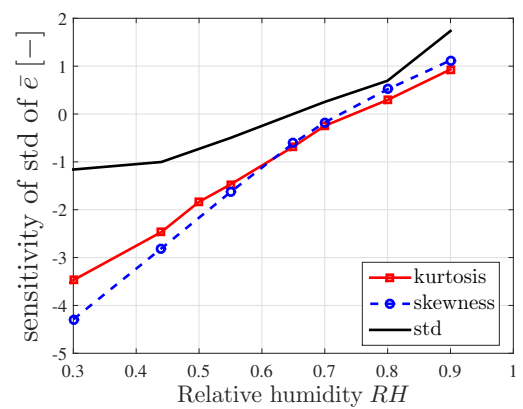

(b)

Figure 13. Sensitivity analysis for the case of pair b: the local derivations of (a) the mean and (b) the standard deviation of the meso-scale apparent adhesion energy with respect to standard deviation (std), skewness, and kurtosis of contacting surfaces B2.

The evolution of the apparent adhesion energies and of their uncertainty: From both experimental results and the numerical predictions, it is observed that the apparent adhesion energies increase with the humidity level and decrease with the roughness. It is deduced that the contribution of the capillary interaction is dominant on the $\mathrm{vdW}$ one in this problem, and that the apparent adhesion energies significantly depend on the topology of the contacting surfaces as expected. In addition, because the interacting areas are smaller for the lower adhesion energies, the lower the adhesion energy the larger its uncertainty. This fact is observed from both numerical predictions and experimental results for pair $\mathrm{b}$ and pair $\mathrm{c}$, see Fig. 15. For pair a, that trend is not significant. It is explained by: (i) the uncertainty of adhesion energies resulting from the random surfaces is small as the apparent adhesion energies and the interacting area are higher for the case of pair a, $\Gamma /\left(2 \gamma_{\mathrm{LG}}\right) \geq 10 \%$ for $R H \geq 60 \%$, compared to the other pairs; and (ii) there are other uncertainty sources whose effects are independent of the contact behavior, e.g. the uncertainty of the geometrical dimensions discussed in the following.

Effect of the uncertainty on geometrical dimensions: As it is observed from Fig. 15, for the low apparent adhesion energies, e.g. $\Gamma /\left(2 \gamma_{\mathrm{LG}}\right) \leq 10 \%$, the uncertainties resulting from the scatter in the geometrical dimensions are less important than the ones from the rough surfaces; however they become dominant for the higher values of apparent energies, e.g. the case of pair a for $R H \geq 60 \%$, since the effective contact areas increase.

Validation of the developed method: We remark that there is an error when estimating PSD functions indirectly using the cutoff self-affine formulation described in Eq. (7), see Tab. I. From the comparison in Tab. I between the experimental surfaces and generated surfaces in terms of the mean radius of the summits and their density, the error is negligible for B2 and Sub1 (involved in pair b), moderate for B1 (involved in pair a), and significant for Sub2 (involved in pair c). Interestingly, the deviations between experimental data and 


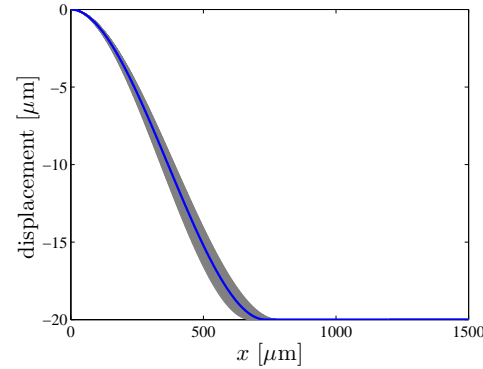

(a)

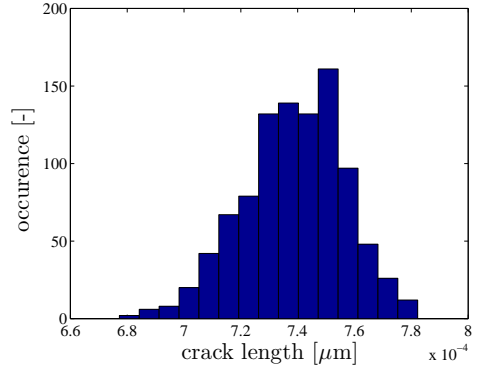

(b)

Figure 14. MCS of the beam subjected to stiction for the case of pair b at $R H=0.65$. (a) 1000 realizations of beam structures with a highlighted one, (b) Histogram of the crack lengths obtained from MCS.

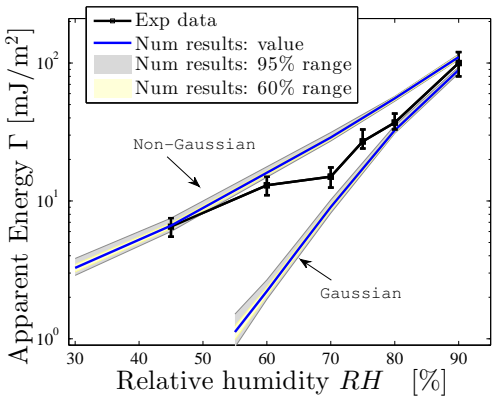

(a)

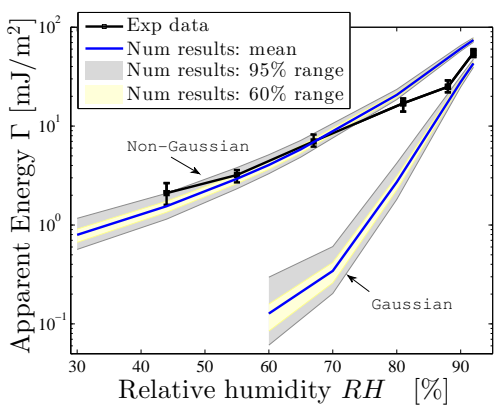

(c)

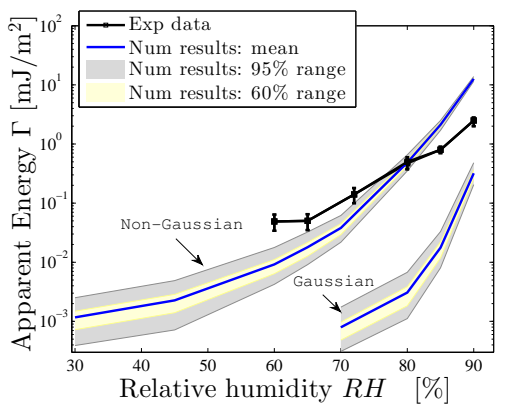

(e)

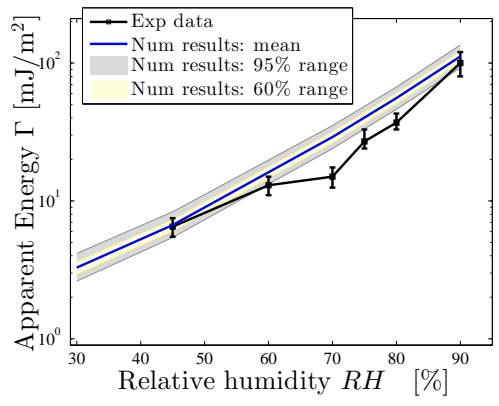

(b)

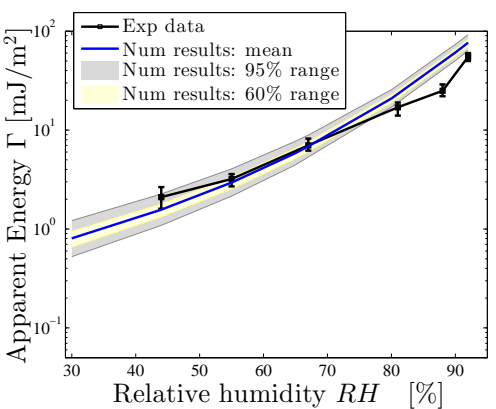

(d)

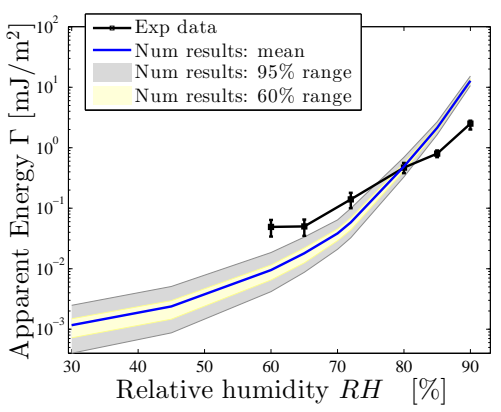

(f)

Figure 15. Comparison in terms of the apparent adhesion energies between experimental (Exp) data and numerical (Num) results. Each row corresponds to one pair: pair a, pair b, pair c (from top to bottom). The numerical results include three cases: (G) Gaussian contacting surfaces, (NG) non-Gaussian contacting surfaces, and (GEO) extension of case (NG) to account for the uncertainty in the geometrical dimensions. The cases (G) and (NG) are illustrated in the first column (a, c, e), while second column, (b, d, f), reports the results for the case (GEO).

numerical results have the same order: small for pair $\mathrm{b}$ (except for $R H=0.88$ ), moderate for pair a, and significant for pair c, see Fig. 15 and Tab. III. Considering the pair c, in comparison with the two other pairs, the reported experimental adhesion energies of pair $\mathrm{c}$ are the lowest ones with values in the range $\Gamma \in[0.035,2.7] \mathrm{mJ} / \mathrm{m}^{2}$ for $R H \in[0.6,0.9]$. Based 
on these values, the ratio between the interacting area and the apparent one is of the order of $\Gamma /\left(2 \gamma_{\mathrm{LG}}\right) \in[0.03 \%, 1.9 \%]$. With such a small part of the topology involved in the contact interaction, the sensitivity on the predicted apparent adhesion energies on the input data including the first order mPDF and the PSD function is significant. Therefore, the errors resulting from the indirect estimation of the PSD function, as discussed previously, lead to an important deviation of the numerical predictions which is observed in Tab. III for pair c.

A remark follows from that comparison: when dealing with contact problems in which the ratios of the interacting areas to the apparent areas are small, e.g $<2 \%$ for the considered cases, a high accuracy is required when estimating the height distribution and the PSD functions. In practice, this could demand an important effort on the whole process including the measurement using AFM on which the size and the sampling length are defined, the processing of AFM data for which the filters are applied, and the numerical characterization of the processed data to obtain the first order mPDF and the PSD function.

\section{CONCLUSION}

Because of the small size of MEMS, adhesive contact can lead to their failure. Furthermore, due to the roughness of the contacting surfaces and the short range of the adhesive stress (nanometers), there exists an uncertainty in the behavior of microstructures subjected to adhesion. To evaluate the probabilistic MEMS behaviors, we have developed a stochastic modelbased multi-scale method in which the effect of the nonGaussian heights distribution of the contacting surfaces was accounted for. The model consists in: (i) the characterization and simulation of the non-Gaussian random surfaces, and (ii) the propagation of the randomness of the contacting surfaces through a multi-scale FE model to quantify the uncertainty in the structural behaviors. The model was also extended to account for the randomness of the geometry dimensions. With the developed model, the stiction risk of a micro-structure can be investigated in a probabilistic way at its design stage.

The developed model was verified with experimental data of cantilever beam stiction tests reported in the literature. Although we do not have direct access to the AFM measurements of surfaces to implement accurately the characterization step, the evaluation of the adhesion energies with their uncertainties in terms of roughness and humidity level are well predicted. Using the numerical model, the following observations are pointed out and verified by the experimental results:

(i) Non-Gaussianity of contacting surfaces is an important factor in predicting stiction risk. Since the contacting area is small in comparison with the apparent one, and since the contact involves only the highest asperities, the tail of the surface heights distribution plays a significant role in the adhesive contact. Using the ME method, the first order mPDF of the contact surface heights was estimated accounting for the non-Gaussian characteristics. For the studied cases, the apparent adhesion energies are increased compared to the cases of Gaussian distribution because of the negative skewness. (ii) Uncertainty is an important aspect of stiction risk. Because the number of contacting asperities are smaller for the lower apparent adhesion energies, the smaller these energies the larger their uncertainties. The effect of random surfaces on the probabilistic adhesion behaviors is important for the low apparent adhesion energies, e.g $<10 \%$ of the theoretical one for the considered cases, and becomes negligible in comparison with the one due to the geometry dimensions uncertainty for the higher energies.

(iii) The numerical investigation shows that the smaller the adhesion energies the higher the accuracy required when characterizing the random surfaces by the first order $\mathrm{mPDF}$ and the PSD function.

In the deterministic cases, the contact behaviors, including friction coefficient [10], do not depend on the nominal contact area but on the applied forces. However, the model developed in this work shows that uncertainties arise when the effective contact area involved during stiction is not sufficiently large to obtain a statistically representative contact behavior: the smaller the nominal surface area, the larger the uncertainty in the adhesive contact forces. The model thus partly answers the question raised in [26]: “... why one MEMS device sticks and another identical one does not"

In a future work, other experiments with a direct access to AFM measures of the surfaces involved during the stiction tests are required in order to further validate the model. Furthermore, experiments are mandatory to identify the relation between statistical properties of surfaces and their fabrication method. Combining such a Process-Structure-Properties (PSP) linkage to our stochastic multi-scale model of stiction would allow optimizing the fabrication process with respect to an accepted stiction risk.

Finally the model developed in this paper does not account for irreversible processes, such as plasticity and damage [51], [52], [4], [53], which affect the surface properties, hence the stiction behavior, in particular under cyclic working conditions. These aspects will be investigated in a future work by substituting the asperity model considered in this work by a more evolved semi-analytical micro-mechanical model of surface interaction, e.g. based on a combination of conjugate gradient method, discrete convolution and Fast Fourier Transform allowing elasto-plasticity [54], [55] and/or adhesion [56] to be accounted for. However when considering cyclic loading, the constant Laplace pressure assumption in the meniscus might also have to be modified following the argumentation in [29], [47].

\section{APPENDIX A}

\section{EVALUATION OF THE IRREVERSIBLE BEHAVIORS}

\section{A. Development of a criterion}

When considering the contact problem between a sphere of radius $R$ and a flat surface, the maximum stress predicted by Hertz model is

$$
\sigma_{\max }=\frac{2}{\pi} E^{\prime} \sqrt{\frac{\delta}{R}},
$$

where $E^{\prime}=\left[\left(1-\nu_{1}^{2}\right) / E_{1}+\left(1-\nu_{2}^{2}\right) / E_{2}\right]^{-1}$, and $\delta$ is the interference. Irreversible behavior can thus be assessed with 
the work of Tabor [57] stating that the onset of plastic or irreversible deformation occurs when $\sigma_{\max }>0.6 \sigma_{H}$, where $\sigma_{H}$ is the material hardness. To assess the importance of plastic deformation in the rough surfaces contact problems, one can examine the ratio $A_{P} / A$, where $A$ is the total physical contact area of all contacting asperities, and $A_{P}$ is the physical contact area of the asperities such that $\sigma_{\max }>0.6 \sigma_{H}$.

In their work, Greenwood and Williamson [9] have shown that this ratio mainly depends on a plasticity index $\Psi_{P}$ and that when $\Psi_{P}<0.6$, plastic deformation is negligible since $A_{P} / A<0.02$, while for $\Psi_{P}>1$, plasticity becomes dominant since $A_{P} / A>0.5$. In this work, since $\mathrm{GW}$ model is not applied, the plasticity index cannot be used and we evaluate the ratio $A_{P} / A$ directly from the semi-analytical contact model.

\section{B. Application to the considered surfaces}

The maximum stress (14) for each identified asperity for the surfaces of pairs a, b, and c, see Tab. I, is illustrated in Fig 16. In these figures, we consider contact distances $\bar{d}$ well below the adhesive part of the contact curves, see Fig. 12, in order to consider a worst-case scenario. The hardness of (poly)silicon is $\sigma_{H}=12.8 \mathrm{GPa}$. As it is observed from Fig 16, in all the cases, the maximum stresses are smaller than $0.6 \sigma_{H}=7.7 \mathrm{GPa}$. In other words, the areas $A_{P}$ are zero. Therefore, the negligibility of the irreversible deformation is confirmed.

\section{APPENDIX B}

\section{STOCHASTIC MODEL OF RANDOM MESO-SCALE APPARENT CONTACT FORCES.}

In this appendix, the development of the stochastic model is summarized from our previous work [30], and then implemented for the stiction model reported in Sec. IV. The stochastic model is constructed using the generalized polynomial chaos expansion (gPCE) method [58], [59], [60]. The input data is a set of $m$ explicitly evaluated apparent contact forces, from which the set of $m$ corresponding physical parameters vectors $\left\{\mathbf{v}^{(1)}, \ldots, \mathbf{v}^{(m)}\right\}$ - samples of the random vector $\mathbf{V}$ is obtained using the parametrization process described in Section III-B1. The value $m$ is chosen to satisfy the condition that the distribution of the random vector $\mathbf{V}$ evaluated from these input data has converged. The stochastic model is constructed in two steps: (i) data processing and (ii) construction of the stochastic model using gPCE on the processed data.

\section{A. Input data processing}

There are two procedures applied on the input data, introduction of the physical constraints and dimension reduction, explained in the following.

1) Introduction of physical constraints: There are three physical constraints applied on the vector $\mathbf{v}$ : (i) $\bar{e} \geq 0$, (ii) $\bar{f}_{\max } \geq 0$; and (ii) $\bar{d}_{\max }>\bar{d}_{\text {limit }}$, see Fig. 5. In order to precondition these constraints, we introduce a standardized

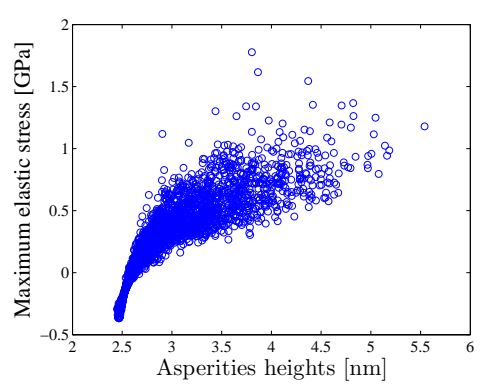

(a)

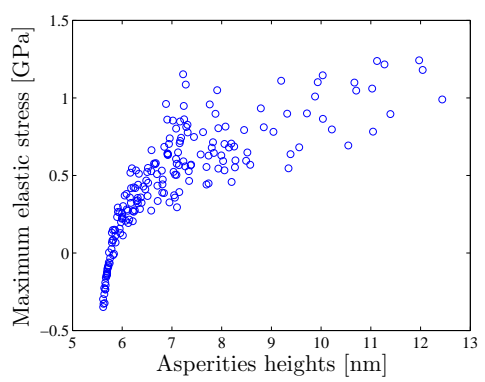

(b)

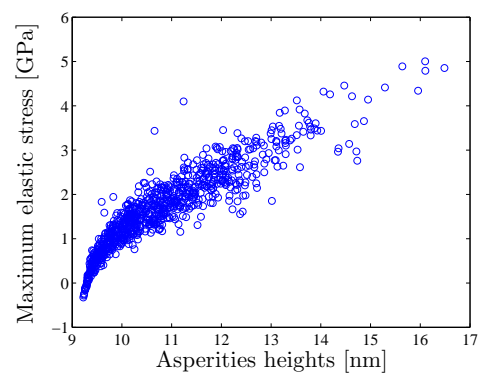

(c)

Figure 16. The maximum elastic stress evaluated by Eq. (14) for the identified contacting asperities in the cases: (a) pair a with contact distance $\bar{d}=2.2 \mathrm{~nm}$; (b) pair b with contact distance $\bar{d}=5.7 \mathrm{~nm}$; (c) pair c with contact distance $\bar{d}=9 \mathrm{~nm}$.

random vector $\mathbf{Q}$ representing the random vector $\mathbf{V}$ whose samples $\left\{\mathbf{q}^{(1)}, \ldots, \mathbf{q}^{(m)}\right\}$ are evaluated as

$$
\begin{aligned}
& \mathbf{q}^{(k)}=\left[\frac{\log \left(\bar{e}^{(k)}\right)}{\sigma_{\log (\bar{e})}}, \frac{\log \left(\bar{f}_{\text {max }}^{(k)}\right)}{\sigma_{\log \left(\bar{f}_{\max }\right)}}, \frac{\bar{d}_{\text {max }}^{(k)}}{\sigma_{\bar{d}_{\max }}}, \frac{\log \left(\bar{d}_{\text {max }}^{(k)}-\bar{d}_{\text {limit }}^{(k)}\right)}{\sigma_{\log \left(\bar{d}_{\max }-\bar{d}_{\text {limit }}\right)}}\right]^{\mathrm{T}}, \\
& \text { with } k=1, \ldots, m \text {, }
\end{aligned}
$$

where $\sigma$. is the standard deviation of the random variable $\cdot$ Note that the random vector $\mathbf{Q}$ is free from physical constraints and valued in $\mathfrak{R}^{4}$. With this mathematical formulation, the random vector $\mathbf{V}$ evaluated from random vector $\mathbf{Q}$ by using the inversion of Eq. (15) automatically respects the physical bounds.

2) Linear dimension reduction: The linear dimension reduction technique [61], [62], [63] is applied to reduce the effect of the curse of dimensionality. Applying the principal component transformation, we deduce the random vector $\mathbf{H}$ with its realization $\boldsymbol{\eta}$ evaluated by

$$
\boldsymbol{\eta}^{\mathrm{T}}=(\mathbf{q}-\overline{\mathbf{q}})^{\mathrm{T}}[A][\Lambda]^{-1 / 2}
$$

where $[A]=\left[\mathbf{a}_{1}, \ldots, \mathbf{a}_{4}\right]$ is a normalized matrix of the 4 eigenvectors of the covariance matrix $\left[C_{\mathbf{Q}}\right],[\Lambda]=\operatorname{diag}(\boldsymbol{\lambda})$ is 
a diagonal matrix of the 4 corresponding ordered eigenvalues $\lambda_{1} \geq \ldots \geq \lambda_{4} \geq 0$, and $\overline{\mathbf{q}}$ is the mean vector. Mean vector $\overline{\mathbf{q}}$ and covariance matrix $\left[C_{\mathbf{Q}}\right]$ are evaluated from the samples $\left\{\mathbf{q}^{(1)}, \ldots, \mathbf{q}^{(m)}\right\}$. Thanks to the arrangement of the eigenvalues, the following approximation is achieved:

$$
q_{i} \simeq \bar{q}_{i}+\sum_{j=1}^{N_{g}} \sqrt{\lambda_{j}} A_{i j} \eta_{j}, \quad \text { for } i=1, \ldots, 4 \text { and } N_{g} \leq 4
$$

That approximation implies that the random vector $\mathbf{Q}$ can be represented by the $N_{g}$-dimensional random vector $\widetilde{\mathbf{H}}$ with its realizations $\widetilde{\boldsymbol{\eta}}$ defined by the first $N_{g}$ components of the vector $\eta$

$$
\widetilde{\boldsymbol{\eta}}=\left\{\eta_{1}, \ldots, \eta_{N_{g}}\right\}
$$

\section{B. Stochastic model}

There exists a mapping from the random vector $\widetilde{\mathbf{H}}$ to the random vector $\mathbf{V}$ using the sequence of Eqs. $(17,15)$. Therefore, the stochastic model is constructed on the random vector $\widetilde{\mathbf{H}}$ as a truncated $N_{d}$-order gPCE formulated as

$$
\underbrace{\widetilde{\mathbf{H}}^{\mathrm{PC}}=\sum_{\alpha=1}^{N} \mathbf{c}_{\alpha} \Psi_{\alpha}(\boldsymbol{\Xi})}_{N_{d} \text {-order gPCE }} \stackrel{\text { d. }}{\approx} \widetilde{\mathbf{H}},
$$

where $\stackrel{\mathrm{d}}{\approx}$ means the approximation in terms of distribution, $\mathbf{c}_{1}, \ldots, \mathbf{c}_{N}$ are vectors of coefficients in $\mathfrak{R}^{N_{g}}$ to be identified, and where $\Psi_{1}(\boldsymbol{\Xi}), \ldots, \Psi_{N}(\boldsymbol{\Xi})$ are the renumbered orthogonal Legendre polynomials defined in $\boldsymbol{\Xi} \in[0,1]^{N_{g}}$ and whose orders are lower than or equal to $N_{d}$. The number of polynomials in this expansion is $N=\left(N_{d}+N_{g}\right) ! / N_{g} ! N_{d} !$.

The coefficients $\mathbf{c}_{1}, \ldots, \mathbf{c}_{N}$ are identified in order to ensure the approximation in terms of distribution as stated by Eq. (19). The identification of these coefficients was developed in [30] following two steps: (i) estimating the conditional CDFs of the random vector $\widetilde{\mathbf{H}}$ from its $m$ samples $\left\{\widetilde{\boldsymbol{\eta}}^{(1)}, \ldots, \widetilde{\boldsymbol{\eta}}^{(m)}\right\}$ by using kernel density estimation, and (ii) projecting the inverse functions of these CDFs on the Legendre polynomials to evaluate the gPCE coefficients.

\section{Implementation of the stochastic model for the stiction model reported in $\mathrm{Sec}$. IV}

The stochastic model of the apparent contact forces is implemented for the stiction test explained in Sec. IV. The value of $m$ is chosen based on a convergence analysis of the PDF of the random vector V, see Fig. 17(a) for the case of apparent adhesion energy $\bar{e}$. From that analysis, we chose $m=5000$. From the $m$ input samples of the random vector $\mathbf{V}$, the data processing including the enforcement of physical constraints and the dimension reduction is performed. The reduced dimension number $N_{g}$ is identified through the investigation of the error given by $\operatorname{Err}^{\mathrm{DR}}\left(N_{g}\right)=\frac{\lambda_{N_{g}+1}+\cdots+\lambda_{4}}{\lambda_{1}+\cdots+\lambda_{4}}$. As it is observed from Fig. 17(b), the reduced dimension number can be chosen as three. The gPCE model is developed for the random vector $\widetilde{\mathbf{H}}$ resulting from the dimension reduction process. As it is observed from the convergence of the gPCE model illustrated in Fig. 18, the 12th-order gPCE model approximates accurately the distribution of the input data.

From the generated samples of the uniformly distributed random vector $\boldsymbol{\Xi}$, one can calculate the corresponding samples of the random vector $\widetilde{\mathbf{H}}$ using the converged gPCE model, and then evaluate the corresponding samples $\mathbf{v}$ of the random vector $\mathbf{V}$ using the sequence Eqs. $(17,15)$. From these samples, the corresponding apparent adhesive contact forces are constructed using Eq. (12), see Fig. 12(c, f, i) for their illustration.

\section{APPENDIX C}

\section{IDENTIFICATION OF THE ELEMENT LENGTH AND OF THE} MESO-SCALE LENGTH FOR THE APPLICATION REPORTED IN SEC. IV

The element length $l_{e}$ and the meso-scale length $l^{\text {meso }}$ are identified using a convergence analysis. Toward this end, we consider the deterministic problem for which the adhesive behavior between the beam bottom surface and the substrate is uniform. The adhesive behavior is modeled using one realization, illustrated in Fig. 19(a), of the random apparent contact forces. The FE method developed in Sec. III-C is applied to evaluate the crack length and upper-scale apparent adhesion energy $\Gamma$ given by Eq. (13). We note that for this deterministic problem, the upper scale apparent adhesion energy $\Gamma$ is the adhesion energy $\bar{e}$ of the apparent adhesive contact force, see Fig. 5. The convergence analysis is achieved by comparing the FE prediction with the adhesion energy $\bar{e}$, as illustrated in Fig. 19(b). We can chose the element length and the mesoscale length as $l_{e}=7.5 \mu \mathrm{m}$ and $l^{\text {meso }}=1.5 \mu \mathrm{m}$ as they lead to a numerical prediction with a difference of $2.5 \%$ compared to the analytical ones.

This analysis confirms that with $l^{\text {meso }}=1.5 \mu \mathrm{m}$ the variation of the central beam for the distance $l^{\text {meso }}$ is negligible in comparison with the range of the adhesive behavior. In Fig. 19(c), the ACFs of the considered surfaces, see Tab. I, are illustrated. The identified value of $l^{\text {meso }}$ is bigger than the correlation lengths at which the ACFs of the considered surfaces almost vanish, $\sim 0.3[\mu \mathrm{m}]$.

\section{REFERENCES}

[1] J. A. Knapp and M. P. de Boer, "Mechanics of microcantilever beams subject to combined electrostatic and adhesive forces," Journal of Microelectromechanical Systems, vol. 11, no. 6, pp. 754-764, 2002.

[2] S. J. Timpe and K. Komvopoulos, "An experimental study of sidewall adhesion in microelectromechanical systems," Journal of microelectromechanical systems, vol. 14, no. 6, pp. 1356-1363, 2005.

[3] W. M. Van Spengen, R. Puers, and I. De Wolf, "A physical model to predict stiction in MEMS," Journal of Micromechanics and Microengineering, vol. 12, no. 5, p. 702, 2002.

[4] Y.-P. Zhao, L. S. Wang, and T. Yu, "Mechanics of adhesion in MEMS - a review," Journal of Adhesion Science and Technology, vol. 17, no. 4, pp. 519-546, 2003. [Online]. Available: https://doi.org/10.1163/15685610360554393

[5] X. Xue, A. A. Polycarpou, and L. M. Phinney, "Measurement and modeling of adhesion energy between two rough microelectromechanical system (MEMS) surfaces," Journal of Adhesion Science and Technology, vol. 22, no. 5-6, pp. 429-455, 2008.

[6] M. de Boer, "Capillary adhesion between elastically hard rough surfaces," Experimental Mechanics, vol. 47, pp. 171-183, 2007. [Online]. Available: http://dx.doi.org/10.1007/s11340-006-0631-z 


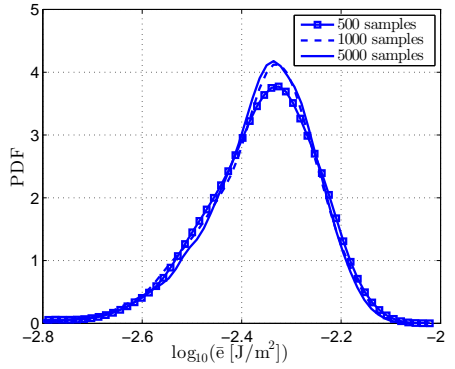

(a)

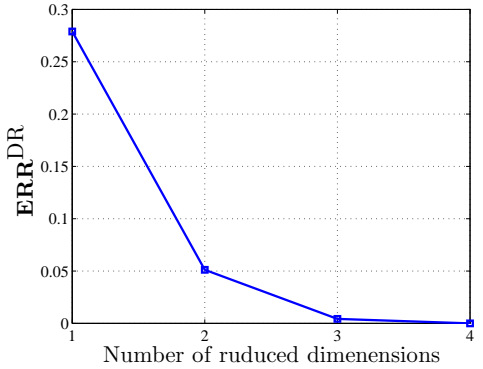

(b)

Figure 17. The convergence analyses: (a) with respect to $m$ when approximating the PDF of the apparent adhesion energy $\bar{e}$, (b) with respect to the error Err ${ }^{\mathrm{DR}}$ when performing the dimension reduction process.

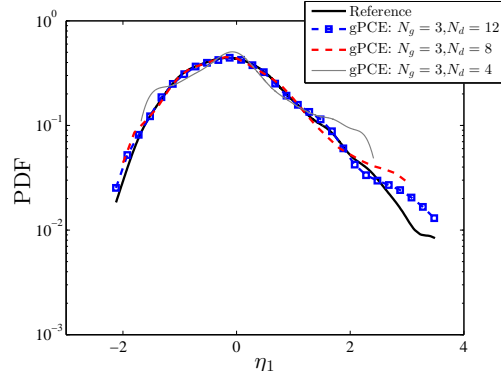

(a)

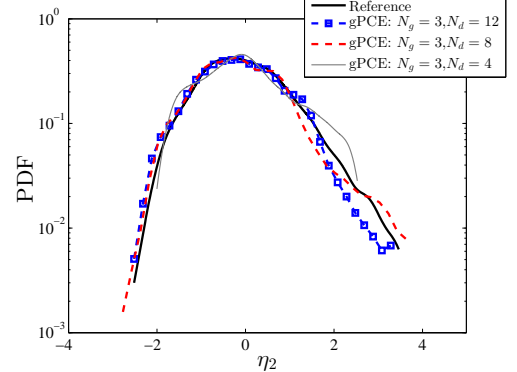

(b)

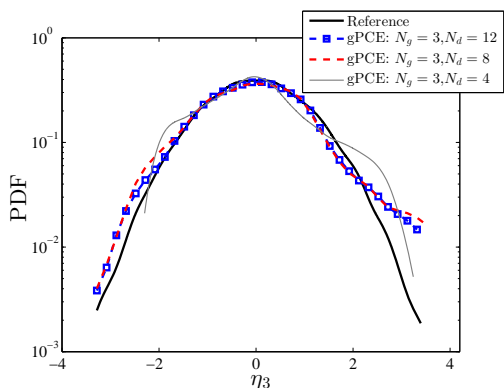

(c)

Figure 18. The convergence analysis of gPCE model described by Eq. (19) in terms of the order $N_{d}$. The figures illustrate the comparisons in terms of the marginal distributions of $\eta_{1}$ (a), $\eta_{2}$ (b), and $\eta_{3}$ (c), between the random vector $\widetilde{\mathbf{H}}^{\mathrm{PC}}$ evaluated using the gPCE model for different values of $N_{d}$ and the reference ones evaluated from the $m$ explicitly evaluated contact forces.

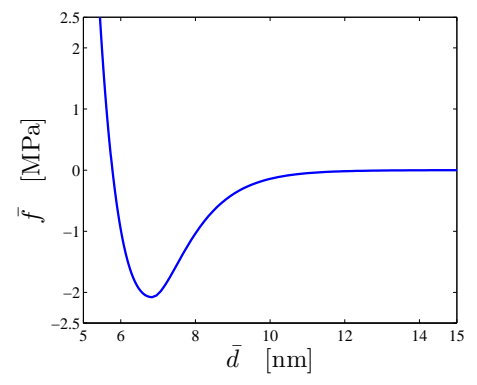

(a)

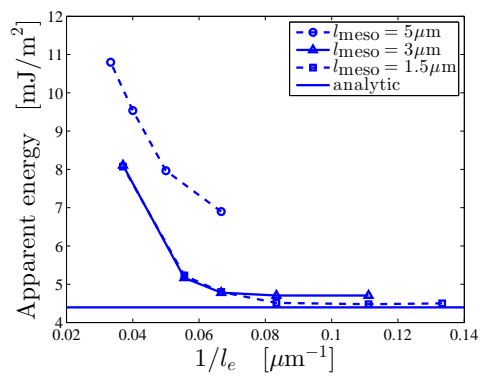

(b)

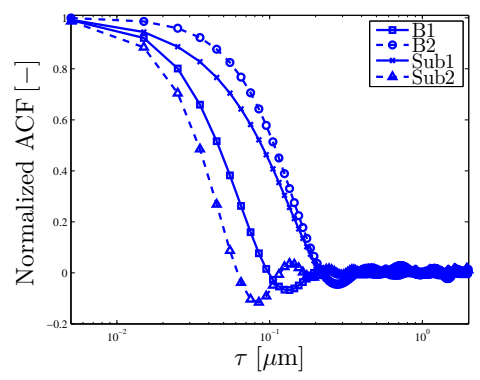

(c)

Figure 19. Convergence analysis of element length $l_{e}$ and meso-scale length $l^{\text {meso }}$ of the FE model. (a) The deterministic contact law extracted from the evaluated apparent contact forces of pair b at $R H=0.65$, see Fig. 12(e). (b) The convergence analysis of $l_{e}$ and $l^{\text {meso }}$ in terms of the upper-scale apparent adhesion energy for the cantilever beam. (c) Normalized ACF of generated surfaces discussed in Sec. IV.

[7] J. W. Rogers, T. J. Mackin, and L. M. Phinney, "A thermomechanical model for adhesion reduction of MEMS cantilevers," Journal of Microelectromechanical Systems, vol. 11, no. 5, pp. 512-520, 2002.

[8] E. Soylemez and M. De Boer, "Capillary-induced crack healing between surfaces of nanoscale roughness," Langmuir, vol. 30, no. 39, pp. 11625 $11633,2014$.

[9] J. A. Greenwood and J. B. P. Williamson, "Contact of nominally flat surfaces," Proceedings of the Royal Society of London. Series A. Mathematical and Physical Sciences, vol. 295, no. 1442, pp. 300-319, 1966.

[10] J. F. Archard, "Elastic deformation and the laws of friction," Proceedings of the Royal Society of London. Series A. Mathematical and Physical Sciences, vol. 243, no. 1233, pp. 190-205, 1957. [Online]. Available: http://rspa.royalsocietypublishing.org/content/243/1233/190.abstract

[11] K. L. Johnson, Contact mechanics. Cambridge university press, 1987.

[12] A. Hariri, J. Zu, and R. B. Mrad, "Modeling of wet stiction in microelectromechanical systems (MEMS)," Journal of microelectromechanical systems, vol. 16, no. 5, pp. 1276-1285, 2007.
[13] R. Ardito, A. Frangi, F. Rizzini, and A. Corigliano, "Evaluation of adhesion in microsystems using equivalent rough surfaces modeled with spherical caps," European Journal of Mechanics - A/Solids, vol. 57, pp. 121-131, 2016. [Online]. Available: http://www.sciencedirect.com/science/article/pii/S0997753815001667

[14] H. Hertz, "Ueber die berührung fester elastischer körper." Journal für die reine und angewandte Mathematik, vol. 92, pp. 156-171, 1882. [Online]. Available: http://eudml.org/doc/148490

[15] B. Derjaguin, V. Muller, and Y. Toporov, "Effect of contact deformations on the adhesion of particles," Journal of Colloid and Interface Science, vol. 53, no. 2, pp. 314 - 326, 1975. [Online]. Available: http://www.sciencedirect.com/science/article/pii/0021979775900181

[16] K. L. Johnson, K. Kendall, and A. D. Roberts, "Surface energy and the contact of elastic solids," Proceedings of the Royal Society of London. A. Mathematical and Physical Sciences, vol. 324, no. 1558, pp. 301-313, 1971. [Online]. Available: http://rspa.royalsocietypublishing.org/content/324/1558/301.abstract

[17] D. Maugis, "Adhesion of spheres: The JKR-DMT transition using 
a dugdale model," Journal of Colloid and Interface Science, vol. 150, no. 1, pp. 243 - 269, 1992. [Online]. Available: http://www.sciencedirect.com/science/article/pii/002197979290285T

[18] R. Ardito, A. Corigliano, A. Frangi, and F. Rizzini, "Advanced models for the calculation of capillary attraction in axisymmetric configurations," European Journal of Mechanics A/Solids, vol. 47, pp. 298 - 308, 2014. [Online]. Available: http://www.sciencedirect.com/science/article/pii/S0997753814000679

[19] R. Ardito, A. Corigliano, and A. Frangi, "Modelling of spontaneous adhesion phenomena in micro-electro-mechanical systems," European Journal of Mechanics - A/Solids, vol. 39, pp. $144-152,2013 . \quad$ [Online]. Available: http://www.sciencedirect.com/science/article/pii/S0997753812001489

[20] R. Ardito, L. Baldasarre, A. Corigliano, B. De Masi, A. Frangi, and L. Magagnin, "Experimental evaluation and numerical modeling of adhesion phenomena in polysilicon MEMS," Meccanica, vol. 48, no. 8, pp. 1835-1844, Oct 2013. [Online]. Available: https://doi.org/10.1007/s11012-013-9767-y

[21] L. Pei, S. Hyun, J. Molinari, and M. O. Robbins, "Finite element modeling of elasto-plastic contact between rough surfaces," Journal of the Mechanics and Physics of Solids, vol. 53, no. 11, pp. 2385 - 2409, 2005. [Online]. Available: http://www.sciencedirect.com/science/article/pii/S0022509605001225

[22] V. A. Yastrebov, J. Durand, H. Proudhon, and G. Cailletaud, "Rough surface contact analysis by means of the finite element method and of a new reduced model," Comptes Rendus Mécanique, vol. 339, pp. 473 - 490, 2011. [Online]. Available: http://www.sciencedirect.com/science/article/pii/S163107211100091X

[23] P. Wriggers, T. V. Van, and E. Stein, "Finite element formulation of large deformation impact-contact problems with friction," Computers \& Structures, vol. 37, no. 3, pp. 319 - 331, 1990. [Online]. Available: http://www.sciencedirect.com/science/article/pii/004579499090324U

[24] B. Luan and M. O. Robbins, "Contact of single asperities with varying adhesion: comparing continuum mechanics to atomistic simulations," Physical Review E, vol. 74, no. 2, p. 026111, 2006.

[25] P.-R. Cha, D. J. Srolovitz, and T. Kyle Vanderlick, "Molecular dynamics simulation of single asperity contact," Acta Materialia, vol. 52, no. 13, pp. 3983 - 3996, 2004. [Online]. Available: http://www.sciencedirect.com/science/article/pii/S1359645404002769

[26] W. M. van Spengen, "A physical model to describe the distribution of adhesion strength in MEMS, or why one MEMS device sticks and another identical one does not," Journal of Micromechanics and Microengineering, vol. 25, no. 12, p. 125012, 2015. [Online]. Available: http://stacks.iop.org/0960-1317/25/i=12/a=125012

[27] E. Soylemez and M. De Boer, "Van der waals force-induced crack healing in dry rough interfaces," Journal of Physics D: Applied Physics, vol. 49, no. 7, 2016.

[28] E. Soylemez and M. P. de Boer, "Crack healing between rough polycrystalline silicon hydrophilic surfaces in n-pentanol and water vapors," Tribology Letters, vol. 59, no. 1, pp. 1-12, 2015.

[29] E. Soylemez and M. De Boer, "Capillary-induced crack healing between surfaces of nanoscale roughness," Langmuir, vol. 30, no. 39, pp. 11625 11633,2014

[30] T.-V. Hoang, L. Wu, S. Paquay, J.-C. Golinval, M. Arnst, and L. Noels, "A computational stochastic multiscale methodology for MEMS structures involving adhesive contact," Tribology International, vol. 110, pp. 401 - 425, 2017. [Online]. Available: http://www.sciencedirect.com/science/article/pii/S0301679X1630370X

[31] T.-V. Hoang, L. Wu, S. Paquay, A. Obreja, R. Voicu, R. Müller, J.C. Golinval, and L. Noels, "A probabilistic model for predicting the uncertainties of the humid stiction phenomenon on hard materials," Journal of Computational and Applied Mathematics, vol. 289, pp. 173195, 2015

[32] S. Chilamakuri and B. Bhushan, "Contact analysis of non-gaussian random surfaces," Proceedings of the Institution of Mechanical Engineers, Part J: Journal of Engineering Tribology, vol. 212, no. 1, pp. 19-32, 1998.

[33] N. Yu and A. A. Polycarpou, "Contact of rough surfaces with asymmetric distribution of asperity heights," Transactions of the ASME-F-Journal of Tribology, vol. 124, no. 2, pp. 367-376, 2002.

[34] F. Yamazaki and M. Shinozuka, "Digital generation of non-gaussian stochastic fields," Journal of Engineering Mechanics, vol. 114, no. 7 pp. 1183-1197, 1988

[35] B. Puig, F. Poirion, and C. Soize, "Non-gaussian simulation using hermite polynomial expansion: convergences and algorithms," Probabilistic Engineering Mechanics, vol. 17, no. 3, pp. 253-264, 2002.
[36] F. Poirion and C. Soize, "Numerical methods and mathematical aspects for simulation of homogeneous and non homogeneous gaussian vector fields," in Probabilistic Methods in Applied Physics, ser. Lecture Notes in Physics, P. Krée and W. Wedig, Eds. Springer Berlin Heidelberg, 1995, vol. 451, pp. 17-53. [Online]. Available: http://dx.doi.org/10.1007/3-540-60214-3-50

[37] E. T. Jaynes, "Information theory and statistical mechanics," Physical review, vol. 106, no. 4, p. 620, 1957

[38] N. Agmon, Y. Alhassid, and R. D. Levine, "An algorithm for finding the distribution of maximal entropy," Journal of Computational Physics, vol. 30 , no. 2, pp. 250-258, 1979.

[39] R. D. Levine and M. Tribus, "Maximum entropy formalism," in Maximum Entropy Formalism Conference (1978: Massachusetts Institute of Technology). Mit Press, 1979.

[40] B. Persson, O. Albohr, U. Tartaglino, A. Volokitin, and E. Tosatti, "On the nature of surface roughness with application to contact mechanics, sealing, rubber friction and adhesion," Journal of Physics: Condensed Matter, vol. 17, no. 1, p. R1, 2004.

[41] P. R. Nayak, "Random process model of rough surfaces," Journal of Tribology, vol. 93, no. 3, pp. 398-407, 1971.

[42] M. de Boer and P. de Boer, "Thermodynamics of capillary adhesion between rough surfaces," Journal of Colloid and Interface Science, vol. 311, no. 1, pp. 171 - 185, 2007. [Online]. Available: http://www.sciencedirect.com/science/article/pii/S0021979707002408

[43] S. Cai and B. Bhushan, "Meniscus and viscous forces during separation of hydrophilic and hydrophobic surfaces with liquid-mediated contacts," Materials Science and Engineering: R: Reports, vol. 61, no. 1, pp. 78106, 2008.

[44] N. Yu and A. A. Polycarpou, "Adhesive contact based on the Lennard-Jones potential: a correction to the value of the equilibrium distance as used in the potential," Journal of Colloid and Interface Science, vol. 278, no. 2, pp. 428 - 435, 2004. [Online]. Available: http://www.sciencedirect.com/science/article/pii/S0021979704005454

[45] L. Wu, L. Noels, V. Rochus, M. Pustan, and J.-C. Golinval, "A micro - macro approach to predict stiction due to surface contact in micro electro-mechanical systems," Journal of Microelectromechanical Systems, vol. 20, no. 4, pp. 976 -990, 2011.

[46] B. Cappella and G. Dietler, "Force-distance curves by atomic force microscopy," Surface science reports, vol. 34, no. 1, pp. 1-104, 1999.

[47] E. Soylemez and M. P. de Boer, "Modeling capillary bridge dynamics and crack healing between surfaces of nanoscale roughness," Journal of Micromechanics and Microengineering, vol. 27, no. 12, p. 125023 , 2017.

[48] J. Reddy, "On locking-free shear deformable beam finite elements," Computer Methods in Applied Mechanics and Engineering, vol. 149, no. 1, pp. 113 - 132, 1997. [Online]. Available: http://www.sciencedirect.com/science/article/pii/S0045782597000753

[49] M. De Boer and T. Michalske, "Accurate method for determining adhesion of cantilever beams," Journal of applied physics, vol. 86, no. 2, pp. 817-827, 1999.

[50] A. Y. Suh and A. A. Polycarpou, "Digital filtering methodology used to reduce scale of measurement effects in roughness parameters for magnetic storage supersmooth hard disks," Wear, vol. 260, no. 4, pp. $538-548,2006$

[51] E. E. Jones, K. D. Murphy, and M. R. Begley, "Mechanical measurements of adhesion in microcantilevers: Transitions in geometry and cyclic energy changes," Experimental Mechanics, vol. 43, no. 3, pp. 280-288, Sep 2003. [Online]. Available: https://doi.org/10.1007/BF02410526

[52] L. Zhang and Y.-P. Zhao, "Adhesion of rough surfaces with plastic deformation," Journal of Adhesion Science and Technology, vol. 18, no. 6, pp. 715-729, 2004. [Online]. Available: https://doi.org/10.1163/156856104839329

[53] L. Wu, J.-C. Golinval, and L. Noels, "A micro-model for elasto-plastic adhesive contact in micro-switches: Application to cyclic loading," Tribology International, vol. 57, pp. 137 - 146, 2013. [Online]. Available: http://www.sciencedirect.com/science/article/pii/S0301679X12002757

[54] D. Nélias, E. Antaluca, and V. Boucly, "Rolling of an elastic ellipsoid upon an elastic-plastic flat," Journal of Tribology, vol. 129, no. 4, pp. 791-800, 2007. [Online]. Available: http://dx.doi.org/10.1115/1.2768078

[55] W. Wayne Chen, Q. Jane Wang, F. Wang, L. M. Keer, and J. Cao, "Three-dimensional repeated elasto-plastic point contacts, rolling, and sliding," Journal of Applied Mechanics, Transactions ASME, vol. 75, no. 2, pp. 0210211-02 102 112, 2008. [Online]. Available: http://dx.doi.org/10.1115/1.2755171

[56] M. Bazrafshan, M. de Rooij, M. Valefi, and D. Schipper, "Numerical method for the adhesive normal contact analysis 
based on a dugdale approximation," Tribology International, vol. 112, no. Supplement C, pp. 117 - 128, 2017. [Online]. Available: https://doi.org/10.1016/j.triboint.2017.04.001

[57] D. Tabor, The hardness of metals. Oxford university press, 2000.

[58] R. G. Ghanem and P. D. Spanos, Stochastic finite elements: a spectral approach. Courier Corporation, 2003.

[59] D. Xiu and G. E. Karniadakis, "The wiener-askey polynomial chaos for stochastic differential equations," SIAM journal on scientific computing, vol. 24, no. 2, pp. 619-644, 2002.

[60] C. Soize and R. Ghanem, "Physical systems with random uncertainties: chaos representations with arbitrary probability measure," SIAM Journal on Scientific Computing, vol. 26, no. 2, pp. 395-410, 2004.

[61] J. E. Jackson, A user's guide to principal components. John Wiley \& Sons, 2005, vol. 587.

[62] I. Jolliffe, Principal component analysis. Wiley Online Library, 2002.

[63] I. K. Fodor, "A survey of dimension reduction techniques," 2002. 